The Herd Behavior Index: a new measure for systemic risk in financial markets

Jan Dhaene, Daniël Linders, Wim Schoutens, David Vyncke

DEPARTMENT OF ACCOUNTANCY, FINANCE AND INSURANCE (AFI) 


\title{
The Herd Behavior Index: a new measure for systemic risk in financial markets
}

\author{
Jan Dhaene* $\quad$ Daniël Linders ${ }^{\dagger} \quad$ Wim Schoutens ${ }^{\ddagger} \quad$ David Vyncke $^{\S}$
}

Version: September 12, 2011

\begin{abstract}
We introduce a new and easy to calculate measure for systemic risk in financial markets. This measure is baptized the Herd Behavior Index (HIX). It is modelindependent and forward looking, based on observed option data.

In order to determine the degree of systemic risk or herd behavior in a financial market one should compare the observed market situation with the extreme (theoretical) situation under which the whole system is driven by a single factor. The Herd Behavior Index (HIX) is defined as the ratio of an option-based estimate of the risk-neutral variance of the market index and an option-based estimate of the corresponding variance of this extreme market situation. Using the theory of comonotonicity, the extreme situation can easily be backed out of the observed option quotes.

The HIX can be determined for any market index provided an appropriate series of vanilla options is traded on this index as well as on its components. As an illustration, we determine historical values of the 30-days implied Herd Behavior Index for the Dow Jones Industrial Average, covering the period January 2003 to October 2009.
\end{abstract}

Keywords: Comonotonicity; systemic risk; correlation; VIX volatility index

\section{Introduction}

"Men, it has been well said, think in herds, it will be seen that they go mad in herds, while they only recover their senses slowly, and one by one." Charles Mackay (1841).

Systemic risk in financial markets has become a major focus of financial players, regulators, policy makers and market supervisors. It captures the danger of a collapse of

\footnotetext{
${ }^{*}$ K.U.Leuven, Leuven, Belgium. Email: Jan.Dhaene@econ.kuleuven.be

${ }^{\dagger}$ K.ULeuven, Leuven, Belgium. Email: Daniel.Linders@econ.kuleuven.be

$\ddagger$ K.U.Leuven, Leuven, Belgium. Email: wim@schoutens.be

§UGent, Gent, Belgium. Email: david.vyncke@UGent.be
} 
the financial system and the devastating consequences for financial markets and society as a whole. Therefore, an objective estimation of the degree of systemic risk is of utmost importance. It may give the different stakeholders an insight and an opportunity to take the necessary actions. This paper contributes to this complicated matter by introducing a measure for the degree of herd behavior (or co-movement) of asset prices in financial markets. Although hereafter we will mainly restrict to stock markets, the proposed measure can be determined for any market index provided an appropriate series of vanilla options are traded on this index as well as on its components.

The volatility of a stock market index is influenced by the volatilities of the index components and by the dependence structure among them. Higher individual volatilities and/or a stronger dependence relation increases the index volatility. A stronger dependence is a sign of less diversification and a higher degree of herd behavior and increased systemic risk. Bubbles and crashes may be explained in terms of strong herd behavior. The tulipmania in the Netherlands in the 17th century, the internet bubble around 19952000 and the US housing bubble which peaked in 2007 are textbook examples of bubbles driven by greed and by strong herd behavior. All these bubbles lead to major crashes in the relevant markets. Crashes in financial markets typically occur when individuals are driven by panic and join the crowd in a rush to get out of the market, leading to dramatic price movements (fire-sales). The late-2000's financial crisis following the US housing bubble is an example of this phenomenon. Strong herd behavior may be intensified by the tendency of investors to buy recent winners and/or sell recent losers.

Although herd behavior is often irrational, having information about its magnitude is significant in that it gives insight in the level of systemic risk in the market and in the degree of diversification that is obtained by investing in the market index. Similar to volatilities, the degree of herd behavior may be changing over time in a random manner, which makes it a hard task to estimate it from past data. Derivative instruments take a forward looking view and their prices contain information on the market participants' perception on the future evolution of the market. A standard approach is to determine the volatility of a stock or a stock index that is implied by today's market prices of traded options. In a somewhat similar way, we will define and investigate a new barometer for measuring the future degree of herd behavior as implied in today's option quotes on individual stocks in combination with option prices on the basket/index composed out of these individual stocks.

The definition of the Herd Behavior Index (HIX) is based on the idea that the market's perception on the degree of herd behavior should be measured by comparing the actual dependence structure between the future stock prices with the comonotonic dependence structure. To be more precise, the HIX is defined as the ratio of an option-based estimate of the risk-neutral variance of the market index and an option-based estimate of the corresponding variance of this extreme market situation. The HIX can be interpreted as a scaled variance index, with a time-dependent scaling factor. Using the VIX-methodology, the current market situation can easily be backed out of the observed index option prices, while using the theory of comonotonicity, the extreme situation can be backed out of the observed stock option quotes.

Calculating the forward looking Herd Behavior Index on a daily (or more frequent) 
basis may be used for 'herd behavior trading'. Intuitively speaking, when the herd behavior index is large, there is not much diversification possible and index options are relatively expensive compared to the individual stock options. Therefore, a high herd behavior index might suggest to buy individual options and sell index options. The position can then be profitably closed when the market relaxes. One of the advantages of using the concept of comonotonicity to measure the degree of herd behavior is that it allows to specify the optimal portfolio of individual options one should buy in case of a high value of the HIX.

This paper is organised as follows. In Section 2, we describe the financial market assumed throughout this paper. Essential results concerning the theory of comonotonicity that will be used in this paper are recapitulated in Section 3. Using the concept of comonotonicity, the theoretical case of a market with perfect herd behavior is described in that section. In Section 4, we define the HIX and compare it with other possible indices for measuring herd behavior in stock markets. In particular, it will be shown that the HIX outperforms implied correlation as a measure for co-movement. In Section 5, numerical issues concerning the practical calculation of the HIX are considered. In Section 6 we empirically investigate herd behavior by calculating historical HIX-values for the Dow Jones Industrial Average over the period January 2006 - October 2009. Section 7 concludes the paper.

\section{The financial market}

\subsection{Stocks, the market index and options}

We assume a financial market ${ }^{1}$ where $n$ different (dividend or non-dividend paying) stocks, labeled from 1 to $n$, are traded. Current time is 0 , while the time span under consideration is $T$ years. For each stock $i$, its price at time $t, 0 \leq t \leq T$, is denoted by $X_{i}(t)$. Hereafter, we will always silently assume that $X_{i}(t) \geq 0$ for all $i$ and that its first and second order moments are finite. The standard deviation of $X_{i}(t)$ is denoted by $\sigma_{X_{i}(t)}$. Pearson's correlation coefficient between $X_{i}(t)$ and $X_{j}(t)$ is denoted by $\operatorname{corr}\left[X_{i}(t), X_{j}(t)\right]$.

The market index is composed of a linear combination of the $n$ underlying stocks. Denoting the price of the index at time $t$ by $S(t), 0 \leq t \leq T$, we have that

$$
S(t)=w_{1} X_{1}(t)+w_{2} X_{2}(t)+\ldots+w_{n} X_{n}(t),
$$

where $w_{i}, i=1,2, \ldots, n$, are positive weights that are fixed up front. The standard deviation of $S(t)$ is denoted by $\sigma_{S(t)}$.

We assume that market participants have access to a number of European options with maturity T. More precisely, they can trade in European calls and puts on the index and on the individual stocks. We recall that the pay-off at time $T$ of a European call with

\footnotetext{
${ }^{1}$ We use the common approach to describe the financial market via a filtered probability space $\left(\Omega, \mathcal{F},\left(\mathcal{F}_{t}\right)_{0 \leq t \leq T}, \mathbb{P}\right)$, where $\left(\mathcal{F}_{t}\right)_{0 \leq t \leq T}$ represents the history of the market.
} 
maturity $T$ and strike $K$ on the index is given by $(S(T)-K)_{+}$, whereas the pay-off of the corresponding index put option is given by $(K-S(T))_{+}$. The time-0 prices of these index options are denoted by $C[K, T]$ and $P[K, T]$, respectively. Similar pay-offs and notations hold for calls and puts on the constituent stocks. In particular, the time-0 prices of calls and puts on stock $i$ are denoted by $C_{i}[K, T]$ and $P_{i}[K, T]$, respectively.

It is assumed that the financial market is arbitrage-free and that there exists an equivalent risk-neutral pricing measure $\mathbb{Q}$ such that the current price of any pay-off at time $T$ can be represented as the discounted expectation of this pay-off. In this price-recipy, discounting is performed using $r$, which is the continuously compounded time- 0 risk-free interest rate to expiration $T$, whereas expectations are taken with respect to $\mathbb{Q}$. The no-arbitrage condition gives rise to the following expressions for the option prices:

$$
\begin{aligned}
C_{i}[K, T] & =\mathrm{e}^{-r T} \mathrm{E}\left[\left(X_{i}(T)-K\right)_{+}\right], \\
P_{i}[K, T] & =\mathrm{e}^{-r T} \mathrm{E}\left[\left(K-X_{i}(T)\right)_{+}\right],
\end{aligned}
$$

and

$$
\begin{aligned}
C[K, T] & =\mathrm{e}^{-r T} \mathrm{E}\left[(S(T)-K)_{+}\right] \\
P[K, T] & =\mathrm{e}^{-r T} \mathrm{E}\left[(K-S(T))_{+}\right]
\end{aligned}
$$

In formulae (2) and (3), as well as in the remainder of this text, expectations (distributions) of functions of $\left(X_{1}(T), \ldots, X_{n}(T)\right)$ have to be understood as expectations (distributions) under the $\mathbb{Q}$ - measure. We will often call them risk-neutral expectations (distributions). Furthermore, the notations $F_{X_{i}(T)}(x)$ and $F_{S(T)}$ will be used for the time-0 cumulative distribution functions (cdf's) of $X_{i}(T)$ and $S(T)$ under $\mathbb{Q}$.

From here on, in order to avoid unnecessary overloading of the notations, we will omit the fixed time index $T$, when no confusion is possible. For example, we will write $X_{i}$, $C_{i}[K]$ and $F_{X_{i}}(x)$ for $X_{i}(T), C_{i}[K, T]$ and $F_{X_{i}(T)}(x)$, respectively.

\subsection{Risk-neutral stock price distributions}

In practice, only a finite number of strikes are traded for each stock as well as for the index. Therefore, we assume that for stock $i, i=1,2, \ldots, n$, at current time 0 , European call and put options with strikes $0=K_{i, 0}<K_{i, 1}<\ldots<K_{i, m_{i}}$ and maturity $T$ are available in the market. The prices of these options are denoted by $C_{i}\left[K_{i, j}\right]$ and $P_{i}\left[K_{i, j}\right]$, $i=1,2, \ldots, n ; j=0,1, \ldots, m_{i}$. Furthermore, we assume that $F_{X_{i}}^{-1}(1)$ is known and finite. We will denote this 'maximal value' of $X_{i}$ by $K_{i, m_{i}+1}$. In reality, stock and call options may have an unbounded upward potential. However, for numerical reasons, we will enforce a finite upper bound which can be chosen arbitrarily large. The main results that we will derive hereafter will not depend on the choice of the $K_{i, m_{i}+1}$, provided they are chosen sufficiently large. For an optimal choice of the $K_{i, m_{i}+1}$, we refer to Chen et al. (2008). It may happen that only American stock options are available. Note however that an American option price is always larger than or equal to the corresponding European 
option price, which implies that all crucial inequalities that we will derive hereafter remain to hold in that case, see also Section 5.

If option prices $C_{i}[K]$ were available for any strike $K$, we could in principle deduct the implied risk-neutral distribution $F_{X_{i}}$ of the price of stock $i$ at time $T$. However, as we assumed that there are only a finite number of traded strikes on the individual stocks, this distribution $F_{X_{i}}$ is not completely specified. Following Hobson et al. (2005) and Chen et al. (2008), we solve this problem by replacing each $F_{X_{i}}$ by the discrete cdf $\bar{F}_{X_{i}}$, which is defined by

$$
\bar{F}_{X_{i}}(x)=\left\{\begin{array}{cc}
0, & \text { if } x<0, \\
1+\mathrm{e}^{r T} \frac{C_{i}\left[K_{i, j+1}\right]-C_{i}\left[K_{i, j}\right]}{K_{i, j+1}-K_{i, j}}, & \text { if } K_{i, j} \leq x<K_{i, j+1}, j=0,1, \ldots, m_{i}, \\
1, & \text { if } x \geq K_{i, j+1} .
\end{array}\right.
$$

The cdf $\bar{F}_{X_{i}}$ is an empirical version of $F_{X_{i}}$ which arises from approximating the partially known convex call option curve $C_{i}[K]$ by the piecewise linear convex function connecting the observed points $\left(K_{i, j}, C_{i}\left[K_{i, j}\right]\right), j=0,1, \ldots, m_{i}+1$. Denoting this piecewise linear function by $\bar{C}_{i}[K]$, we find $\bar{F}_{X_{i}}$ from the following relation:

$$
\bar{F}_{X_{i}}(x)=1+\mathrm{e}^{r T} \bar{C}_{i}^{\prime}[x+] .
$$

Obviously, any $\bar{C}_{i}[K]$ is an upper bound for the corresponding call option price $C_{i}[K]$ determined by (2). For the traded strikes $K_{i, j}$ however, both values are identical.

The empirical distributions $\bar{F}_{X_{i}}, i=1,2, \ldots, n$, can also be expressed in terms of traded put options prices. Indeed, taking into account the put-call parity

$$
C_{i}[K]+\mathrm{e}^{-r T} K=P_{i}[K]+\mathrm{e}^{-r T} \mathrm{E}\left[X_{i}\right], \quad K \geq 0
$$

we can transform (4) into

$$
\bar{F}_{X_{i}}(x)=\left\{\begin{array}{cc}
0, & \text { if } x<0, \\
\mathrm{e}^{r T \frac{P_{i}\left[K_{i, j+1}\right]-P_{i}\left[K_{i, j}\right]}{K_{i, j+1}-K_{i, j}},} & \text { if } K_{i, j} \leq x<K_{i, j+1}, j=0,1, \ldots, m_{i}, \\
1, & \text { if } x \geq K_{i, j+1} .
\end{array}\right.
$$

Notice that this expression for $\bar{F}_{X_{i}}$ also follows from approximating the partially known convex option curve $P_{i}[K]$ by a fully known piecewise linear function connecting the observed points $\left(K_{i, j}, P_{i}\left[K_{i, j}\right]\right), j=0,1, \ldots, m_{i}+1$. Denoting this piecewise linear function by $\bar{P}_{i}[K]$, we find $\bar{F}_{X_{i}}$ from

$$
\bar{F}_{X_{i}}(x)=\mathrm{e}^{r T} \bar{P}_{i}^{\prime}[x+] .
$$

Remark that the results presented hereafter still hold in case the set of traded strikes are (partially) different for calls and puts, provided one uses the appropriate empirical distributions (4) or (7). This situation is considered in more detail in Linders et al. (2011). Finally, notice that replacing the partially known pricing distributions $F_{X_{i}}$ by the fully specified empirical pricing distributions $\bar{F}_{X_{i}}$ is a prudent strategy in the sense that $\bar{F}_{X_{i}}$ exceeds $F_{X_{i}}$ in convex order. 


\subsection{The volatility of the index}

Let $S$ be the value of the stock market index at time $T$ and $f$ a twice differentiable function on the non-negative real numbers. Then $f(S)$ can be expressed as

$$
\begin{aligned}
f(S) & =f(a)+f^{\prime}(a)\left((S-a)_{+}-(a-S)_{+}\right) \\
& +\int_{0}^{a} f^{\prime \prime}(K)(K-S)_{+} \mathrm{d} K+\int_{a}^{\infty} f^{\prime \prime}(K)(S-K)_{+} \mathrm{d} K,
\end{aligned}
$$

where $a$ is an arbitrary chosen positive real number. From (9) it follows that the pay-off $f(S)$ at time $T$ can be replicated via a static position in pure discount bonds and European options on the index. Indeed, the first term in the right hand side of this expression can be interpreted as the pay-off at time $T$ from a static position in $f(a)$ pure discount bonds, each paying an amount of 1 at time $T$. The other terms can be interpreted as the payoffs from static positions in European calls and puts on the index with maturity $T$. As an example, consider the first integral term which corresponds with a static position in $f^{\prime \prime}(K) \mathrm{d} K$ puts for all strikes $K$ less than $a$.

Consider now the contract of which one leg pays the buyer the pay-off $f(S)$ at time $T$, while the other leg pays the seller a fixed amount $P$ at time $T$, which was agreed upon at the deal's inception. Assuming that $P$ is determined such that the price of the contract is 0 at time 0 , i.e.

$$
0=\mathrm{e}^{-r T} \mathrm{E}[f(S)-P],
$$

and taking expections in (9), leads to the following expression for the forward price $P$ :

$$
\begin{aligned}
P & =\mathrm{E}[f(S)]=f(a)+\mathrm{e}^{r T} f^{\prime}(a)(C[a]-P[a]) \\
& +\mathrm{e}^{r T}\left(\int_{0}^{a} f^{\prime \prime}(K) P[K] \mathrm{d} y+\int_{a}^{\infty} f^{\prime \prime}(K) C[K] \mathrm{d} K\right) .
\end{aligned}
$$

For a proof of (9) and more details on its interpretation, we refer to Carr and Madan (2001) and references therein.

Let us now consider the special case where the function $f$ is given by

$$
f(S)=(S-\mathrm{E}[S])^{2} .
$$

Here, $\mathrm{E}[S]$ is the risk-neutral expected value of the index price at time $T$. It can be interpreted as the time- 0 forward price of the index value at time $T$. Notice that this forward price can be determined at time 0 from the option prices $C[K]$ and $P[K]$ via the put-call parity:

$$
C[K]+\mathrm{e}^{-r T} y=P[K]+\mathrm{e}^{-r T} \mathrm{E}[S], \quad K \geq 0 .
$$

Applying the relations (9) and (11) to the function $f(S)$ defined in (12) and choosing $a=$ $\mathrm{E}[S]$ leads to

$$
(S-\mathrm{E}[S])^{2}=2\left(\int_{0}^{\mathrm{E}[S]}(K-S)_{+} \mathrm{d} K+\int_{\mathrm{E}[S]}^{\infty}(S-K)_{+} \mathrm{d} K\right)
$$


and

$$
\operatorname{Var}[S]=2 \mathrm{e}^{r T}\left(\int_{0}^{\mathrm{E}[S]} P[K] \mathrm{d} K+\int_{\mathrm{E}[S]}^{+\infty} C[K] \mathrm{d} K\right) .
$$

Hence, the risk-neutral variance of the index price at time $T$ can be interpreted as the time-0 forward price of the contract with pay-off $(S-\mathrm{E}[S])^{2}$ at time $T$. Furthermore, the pay-off of this contract can be replicated by a static portfolio consisting of calls and puts on the index.

Notice that a similar interpretation can be found for the variance of the index return $\left(\frac{S-S(0)}{S(0)}\right)$, by choosing

$$
f(S)=\left(\frac{S-S(0)}{S(0)}-\frac{\mathrm{E}[S]-S(0)}{S(0)}\right)^{2}=\left(\frac{S-\mathrm{E}[S]}{S(0)}\right)^{2} .
$$

In this case, the expressions (9) and (11) translate into

$$
\left(\frac{S-\mathrm{E}[S]}{S(0)}\right)^{2}=\frac{2}{S^{2}(0)}\left(\int_{0}^{\mathrm{E}[S]}(K-S)_{+} \mathrm{d} K+\int_{\mathrm{E}[S]}^{\infty}(S-K)_{+} \mathrm{d} K\right)
$$

and

$$
\operatorname{Var}\left[\frac{S-\mathrm{E}[S]}{S(0)}\right]=\frac{2 \mathrm{e}^{r T}}{S^{2}(0)}\left(\int_{0}^{\mathrm{E}[S]} P[y] \mathrm{d} y+\int_{\mathrm{E}[S]}^{+\infty} C[y] \mathrm{d} y\right) .
$$

For notational convenience hereafter we continue with the contract with pay-off (12).

In case the index option prices are known for all strikes, expression (15) can be used to determine the variance of the index in a model-free way, i.e. based on observed option prices without making any model assumption. From here on, we make the more realistic assumption that only a finite number of strikes are traded in the market. Let us denote the first traded put option strike price below $\mathrm{E}[S]$ by $K_{0}$. All traded index put option strikes below $\mathrm{E}[S]$ are denoted by $K_{-i}, i=0,1, \ldots, l$ with $K_{-l}<K_{-l+1}<\ldots<K_{-1}<K_{0} \leq$ $\mathrm{E}[S]$, whereas all traded index call option strikes above $\mathrm{E}[S]$ are denoted by $K_{i}, i=1, \ldots, h$ with $\mathrm{E}[S]<K_{1}<\cdots<K_{h-1}<K_{h}$. Inspired by the methodology that is used for calculating the VIX volatility index, see Chicago Board Options Exchange (2009), we propose the following approximation for the risk-neutral variance of the index price:

$$
\operatorname{Var}[S] \approx 2 \mathrm{e}^{r T} \sum_{i=-l}^{h} \Delta K_{i} Q\left[K_{i}\right]-\left(\mathrm{E}[S]-K_{0}\right)^{2} .
$$

In this approximation, the $\Delta K_{i}$ are related to the resolution of the strike grid. In particular, we have that $\Delta K_{i}=\frac{K_{i+1}-K_{i-1}}{2}$ for $i=-l+1, \ldots, h-1$. For the lowest strike $K_{-l}$ however, $\Delta K_{-l}=K_{-l+1}-K_{-l}$, whereas for the highest strike $K_{h}$, we take $\Delta K_{h}=K_{h}-K_{h-1}$. Furthermore, $Q\left[K_{i}\right]$ is defined as

$$
Q\left[K_{i}\right]=\left\{\begin{array}{cl}
P\left[K_{i}\right], & \text { if } K_{i}<K_{0} \\
\frac{C\left[K_{i}\right]+P\left[K_{i}\right],}{2}, & \text { if } K_{i}=K_{0} \\
C\left[K_{i}\right], & \text { if } K_{i}>K_{0}
\end{array}\right.
$$


The extra term $\left(\mathrm{E}[S]-K_{0}\right)^{2}$ in $(20)$ is a contribution due to the discretization around $\mathrm{E}[S]$. A justification for the approximation used in formula (19) can be found in the appendix. Notice that the approximation (19) for the variance of the index involves all available call option prices at strikes greater than or equal to $K_{0}$ and all put options at strikes lower than or equal to $K_{0}$. Also note that except for the strike $K_{0}$, we did not assume here that traded strikes for puts and call index options are equal.

\section{Perfect herd behavior}

\subsection{Definition}

A subset $A$ of $\mathbb{R}^{n}$ is said to be comonotonic if any pair of elements $\underline{x}$ and $y$ of $A$ are ordered componentwise, i.e. either $x_{i} \leq y_{i}$ for $i=1,2, \ldots, n$, or $x_{i} \geq y_{i}$ for $i=1,2, \ldots, n$ must hold. Intuitively, a comonotonic set is a 'thin' set of which all elements can be ordered from small to large. The random vector $\underline{X}=\left(X_{1}, \ldots, X_{n}\right)$ of the stock prices at time $T$ is said to be comonotonic if it has a comonotonic support, which means that there exists a comonotonic set $A$ such that $\mathbb{P}[A]=1$. Obviously, comonotonicity of $\left(X_{1}, \ldots, X_{n}\right)$ corresponds to an extremal positive dependence structure, where the increase of the outcome of the price of a particular stock $i$ at time $T$, goes hand in hand with an increase of the outcomes of all the other stock prices. This explains why the term comonotonic (common monotonic) is used.

Notice that we defined comonotonicity of $\underline{X}$ in the $\mathbb{P}$ - world. As comonotonicity is defined in terms of the support of $\underline{X}$ and moreover, $\mathbb{P}$ and $\mathbb{Q}$ are equivalent measures, we have that comonotonicity in the $\mathbb{P}$ - world is equivalent with comonotonicity in the $\mathbb{Q}$ - world. For an extensive overview of the theory of comonotonicity, we refer to Dhaene et al. (2002a). Financial and actuarial applications are described in Dhaene et al. (2002b). An updated overview of applications of comonotonicity can be found in Deelstra et al. (2010).

Perfect herd behavior over a $T$ - year time horizon corresponds with a comonotonic dependence structure for the price vector $\underline{X}$, meaning that from today's point of view all stock prices at time $T$ are driven by a single source of randomness: if one stock price will turn out to be large at time $T$, all other stock prices will be large too. In practice, stock markets are typically not comonotonic. Nevertheless, in this section, we pay attention to the comonotonic case, as we will need it for measuring the 'implied degree of herd behavior' in the next section. Indeed, we will measure the degree of herd behavior by comparing an appropriate linear combination of observed index option prices with the corresponding linear combination in the corresponding comonotonic market.

Several characterizations exist for the notion of comonotonicity. In particular, one has that the vector $\underline{X}$ of the stock prices at time $T$ with marginal distributions denoted by $F_{X_{i}}, i=1,2, \ldots, n$, is comonotonic if and only if

$$
\left(X_{1}, \ldots, X_{n}\right) \stackrel{\mathrm{d}}{=}\left(F_{X_{1}}^{-1}(U), \ldots, F_{X_{n}}^{-1}(U)\right)
$$


where $U$ is a uniform $(0,1)$ r.v. and ' $\stackrel{\mathrm{d}}{=}$ ' is used to denote 'equality in distribution'. Furthermore, $F_{X_{i}}^{-1}$ is the usual inverse of the cdf $F_{X_{i}}$. Characterization (21) clearly shows that comonotonic risks are indeed driven by a single (systemic) factor and exhibit extremal herd behavior. As mentioned above, the pricing distributions $F_{X_{i}}$ are in general unknown. Therefore, hereafter, we will often express our results in terms of the empirical distributions $\bar{F}_{X_{i}}$ defined in (4) or (7). In this case, the inverses $\bar{F}_{X_{i}}^{-1}$ are given by

$$
\bar{F}_{X_{i}}^{-1}(p)=K_{i, j} \text { if } \bar{F}_{X_{i}}\left(K_{i, j-1}\right)<p \leq \bar{F}_{X_{i}}\left(K_{i, j}\right), \quad j=0,1, \ldots, m_{i}+1, p \in(0,1),
$$

with $K_{i,-1}=-1$, by convention.

The weighted sum of the components of the comonotonic vector defined in (21) is denoted by $S^{c}$ :

$$
S^{c}=\sum_{i=1}^{n} w_{i} F_{X_{i}}^{-1}(U) .
$$

The distribution of $S^{c}$ is in general unknown. Therefore, we also introduce the comonotonic sum based on the empirical marginal distributions:

$$
\bar{S}^{c}=\sum_{i=1}^{n} w_{i} \bar{F}_{X_{i}}^{-1}(U) .
$$

In the notation $\bar{S}^{c}$, the superscript ' $c$ ' means that the terms in the sum are comonotonic, whereas the bar indicates that the empirical distributions $\bar{F}_{X_{i}}$ are used. Similar notational conventions are made for other symbols that we will introduce hereafter. We will call $\bar{S}^{c}$ the comonotonic index price at time $T$. Taking into account that the cdf's $\bar{F}_{X_{i}}$ are fully known, we find that also the cdf of $\bar{S}^{c}$ is completely specified at current time 0 .

The comonotonic index price $\bar{S}^{c}$ is a synthetically created random variable, the outcome of which will never be observed. Nevertheless, $\bar{S}^{c}$ will turn out to be a usefull random variable because its cdf is the cdf that coincides with the pricing cdf of the value of the stock index at time $T$, provided the pricing distributions of the stock prices $X_{i}$ coincide with the empirical distributions $\bar{F}_{X_{i}}$ and moreover, the stock prices $\left(X_{1}, \ldots, X_{n}\right)$ are comonotonic. In this sense, the cdf of $\frac{X_{2}}{S}$ can be interpreted as the 'extreme cdf' of the stock price index $S$.

Introducing the following notation:

$$
\bar{F}_{X_{i}}^{-1+}(0)=\min _{j}\left\{K_{i, j} \mid \bar{F}_{X_{i}}\left(K_{i, j}\right)>0\right\},
$$

Linders et al. (2011) propose the following algorithm for determining $F_{\bar{S}^{c}}(K)$ :

1. Using (4) or (7), determine all elements of the following set:

$$
A=\left\{\bar{F}_{X_{i}}\left(K_{i, j}\right) \mid i=1, \ldots, n \text { and } j=0,1, \ldots, m_{i}\right\} \backslash\{0\} .
$$

2. With the help of (22), calculate $\sum_{i=1}^{n} w_{i} \bar{F}_{X_{i}}^{-1}(p)$ for all $p \in A$.

3. For any $K \in\left(\sum_{i=1}^{n} w_{i} \bar{F}_{X_{i}}^{-1+}(0), \sum_{i=1}^{n} w_{i} K_{i, m_{i}+1}\right)$, calculate $F_{\bar{S}^{c}}(K)$ from

$$
F_{\bar{S}^{c}}(K)=\max \left\{p \in A \mid \sum_{i=1}^{n} w_{i} \bar{F}_{X_{i}}^{-1}(p) \leq K\right\} .
$$


4. For other values of $K, F_{\bar{S}^{c}}(K)$ is given by

$$
F_{\bar{S}^{c}}(K)=\left\{\begin{array}{cl}
0 & : K<\sum_{i=1}^{n} w_{i} \bar{F}_{X_{i}}^{-1+}(0), \\
\min _{i} \bar{F}_{X_{i}}\left(\bar{F}_{X_{i}}^{-1+}(0)\right) & : K=\sum_{i=1}^{n} w_{i} \bar{F}_{X_{i}}^{-1+}(0), \\
1 & : K>\sum_{i=1}^{n} w_{i} K_{i, m_{i}+1} .
\end{array}\right.
$$

A relevant question that arises is whether it is always possible or not to construct an artificial comonotonic market with pricing ditribution of $\left(X_{1}, \ldots, X_{n}\right)$ given by the dis-

tribution of $\left(\bar{F}_{X_{1}}^{-1}(U), \ldots, \bar{F}_{X_{n}}^{-1}(U)\right)$. This question is considered in Hobson et al. (2005) and Dhaene and Kukush (2010).

\subsection{Characterizations of perfect herd behavior}

We introduce the notations $C^{c}[K]$ and $P^{c}[K]$ related to $S^{c}$ :

$$
\begin{aligned}
& C^{c}[K]=e^{-r T} \mathrm{E}\left[\left(S^{c}-K\right)_{+}\right], \\
& P^{c}[K]=e^{-r T} \mathrm{E}\left[\left(K-S^{c}\right)_{+}\right] .
\end{aligned}
$$

Notice that $C^{c}[K]$ and $P^{c}[K]$ cannot be interpreted as the prices of options that are available in the market, they should only be interpreted as functions of $K$.

The following theorem states a number of equivalent characterizations for stock prices $\left(X_{1}, \ldots, X_{n}\right)$, with $X_{i} \stackrel{\mathrm{d}}{=} F_{X_{i}}^{-1}(U), i=1,2, \ldots, n$, to be comonotonic.

Theorem 1 Consider the vector $\underline{X}=\left(X_{1}, \ldots, X_{n}\right)$ and suppose that the cdf of $X_{i}$ is given by $F_{X_{i}}, i=1,2, \ldots, n$. The following statements are then equivalent:

$$
\left(X_{1}, \ldots, X_{n}\right) \text { is comonotonic. }
$$

$$
S \stackrel{d}{=} S^{c}
$$

$$
\operatorname{Var}[S]=\operatorname{Var}\left[S^{c}\right]
$$

$$
C[K]=C^{c}[K], \quad \text { for all } K \geq 0 \text {. }
$$

$$
P[K]=P^{c}[K], \quad \text { for all } K \geq 0 \text {. }
$$


Proof. The proof of $(1) \Rightarrow(2) \Rightarrow(3)$ is trivial.

In order to prove $(3) \Rightarrow(1)$, notice that the equivalence of the variances of $S$ and $\bar{S}^{c}$ can be expressed as

$$
\sum_{i, j=1}^{n} \operatorname{corr}\left[X_{i}, X_{j}\right] \sigma_{X_{i}} \sigma_{X_{j}}=\sum_{i, j=1}^{n} \operatorname{corr}\left[F_{X_{i}}^{-1}(U), F_{X_{j}}^{-1}(U)\right] \sigma_{X_{i}} \sigma_{X_{j}} .
$$

Taking into account that

$$
\operatorname{corr}\left[X_{i}, X_{j}\right] \leq \operatorname{corr}\left[F_{X_{i}}^{-1}(U), F_{X_{j}}^{-1}(U)\right], \quad i, j=1, \ldots, n,
$$

see equation (72) in Dhaene et al. (2002a), we conclude from the previous equality that

$$
\operatorname{corr}\left[X_{i}, X_{j}\right]=\operatorname{corr}\left[F_{X_{i}}^{-1}(U), F_{X_{j}}^{-1}(U)\right], \quad i, j=1, \ldots, n .
$$

This condition is equivalent to (1), see Theorem 8 in Dhaene et al. (2002a).

The equivalences $(1) \Leftrightarrow(4) \Leftrightarrow(5)$ are immediate consequences of the following well-known equivalence relation:

$$
\begin{aligned}
S \stackrel{\mathrm{d}}{=} S^{c} & \Longleftrightarrow E\left[(S-K)_{+}\right]=E\left[\left(S^{c}-K\right)_{+}\right], \text {for all } K \geq 0 \\
& \Longleftrightarrow E\left[(K-S)_{+}\right]=E\left[\left(K-S^{c}\right)_{+}\right], \text {for all } K \geq 0 .
\end{aligned}
$$

The bivariate special case of (1)-(3) in Theorem 1 can be found in Dhaene et al. (2002a). Moreover, the equivalence of (1) and (2) in Theorem 1 is a special case of a more general result presented in Cheung (2010), who shows that this equivalence remains to hold without assuming the existence of the second order moments.

\subsection{The volatility of the comonotonic index}

As mentioned above, in practice, the option curves $\left\{C_{i}[K] \mid K \geq 0\right\}$ and $\left\{P_{i}[K] \mid K \geq 0\right\}$ are in general not fully known. This observation implies that it is impossible to derive the distribution of $S^{c}$, as well as its related option curves $\left\{C^{c}[K] \mid K \geq 0\right\}$ and $\left\{P^{c}[K] \mid K \geq 0\right\}$ which were defined in (29). Therefore we introduce the quantities $\bar{C}^{c}[K]$ and $\bar{P}^{c}[K]$ related to the comonotonic index $\bar{S}^{c}$ :

$$
\begin{aligned}
& \bar{C}^{c}[K]=\mathrm{e}^{-r T} \mathrm{E}\left[\left(\bar{S}^{c}-K\right)_{+}\right], \\
& \bar{P}^{c}[K]=\mathrm{e}^{-r T} \mathrm{E}\left[\left(K-\bar{S}^{c}\right)_{+}\right] .
\end{aligned}
$$

Somewhat loosely speaking, we will call $\bar{C}^{c}[K]$ and $\bar{P}^{c}[K]$ the prices of the comonotonic index call and put option with strike $K$. Notice that options with pay-offs $\left(\bar{S}^{c}-K\right)_{+}$and $\left(K-\bar{S}^{c}\right)_{+}$are not traded, but as the distribution of $\bar{S}^{c}$ is known, we are able to determine the values of $\bar{C}^{c}[K]$ and $\bar{P}^{c}[K]$. Hereafter, we explain how to determine these values. 
Starting from the expressions (4) for the empirical distributions $\bar{F}_{X_{i}}$, Chen et al. (2008) prove that the comonotonic index call option price $\bar{C}^{c}[K]$ can be expressed as follows:

$$
\bar{C}^{c}[K]=\sum_{i \in N_{K}} w_{i} C_{i}\left[K_{i, j_{i}}\right]+\sum_{i \in \bar{N}_{K}} w_{i}\left(\alpha_{K} C_{i}\left[K_{i, j_{i}}\right]+\left(1-\alpha_{K}\right) C_{i}\left[K_{i, j_{i}+1}\right]\right),
$$

which holds for any $K \in\left(\sum_{i=1}^{n} w_{i} \bar{F}_{X_{i}}^{-1+}(0), \sum_{i=1}^{n} w_{i} K_{i, m_{i}+1}\right)$. In this expression, each $j_{i}$, $i=1,2, \ldots, n$, depends on $K$ and is defined as the unique number contained in the set $\left\{0,1, \ldots, m_{i}+1\right\}$ that satisfies

$$
\bar{F}_{X_{i}}\left(K_{i, j_{i}-1}\right)<F_{\bar{S}^{c}}(K) \leq \bar{F}_{X_{i}}\left(K_{i, j_{i}}\right) .
$$

Notice that $F_{\bar{S}^{c}}(K)$ can be determined using the algorithm presented in Subsection 3.1. Furthermore, the set $N_{K}$ is defined by

$$
N_{K}=\left\{i \in\{1,2, \ldots, n\} \mid \bar{F}_{X_{i}}\left(K_{i, j_{i}-1}\right)<F_{\bar{S}^{c}}(K)<\bar{F}_{X_{i}}\left(K_{i, j_{i}}\right)\right\}
$$

while its complement $\bar{N}_{K}$ is given by

$$
\bar{N}_{K}=\left\{i \in\{1,2, \ldots, n\} \mid F_{\bar{S}^{c}}(K)=\bar{F}_{X_{i}}\left(K_{i, j_{i}}\right)\right\} .
$$

Finally, the coefficient $\alpha_{K}$ in (31) is given by

$$
\alpha_{K}=1-\frac{K-\sum_{i=1}^{n} w_{i} K_{i, j_{i}}}{\sum_{i \in \bar{N}_{K}} w_{i}\left(K_{i, j_{i}+1}-K_{i, j_{i}}\right)} .
$$

One can prove that $\bar{N}_{K}$ is always a non-empty set, so that $\alpha_{K}$ is always well-defined.

From (31) we see that the comonotonic index call options can be considered as synthetically created options, using an appropriately chosen linear combination of traded options on the individual components of the index, with appropriately chosen strikes.

Starting from the expression (7) for the cdf's $\bar{F}_{X_{i}}$, Linders et al. (2011) show that the comonotonic index put option price $\bar{P}^{c}[K]$ is given by

$$
\bar{P}^{c}[K]=\sum_{i \in N_{K}} w_{i} P_{i}\left[K_{i, j_{i}}\right]+\sum_{i \in \bar{N}_{K}} w_{i}\left(\alpha_{K} P_{i}\left[K_{i, j_{i}}\right]+\left(1-\alpha_{K}\right) P_{i}\left[K_{i, j_{i}+1}\right]\right),
$$

which holds for any $K \in\left(\sum_{i=1}^{n} w_{i} \bar{F}_{X_{i}}^{-1+}(0), \sum_{i=1}^{n} w_{i} K_{i, m_{i}+1}\right)$. In this expression for $\bar{P}^{c}[K]$ the indices $j_{i}$ are defined by (32), the sets $N_{K}$ and $\bar{N}_{K}$ by (33) and (34), and the coefficient $\alpha_{K}$ by (35). Notice that (36) can also be determined from the put-call parity applied to index options and stock options, respectively.

Similar to (9) we find the following expression for $f\left(\bar{S}^{c}\right)$ for any twice differentiable function on the non-negative real numbers:

$$
\begin{aligned}
f\left(\bar{S}^{c}\right) & =f(a)+f^{\prime}(a)\left(\left(\bar{S}^{c}-a\right)_{+}-\left(a-\bar{S}^{c}\right)_{+}\right) \\
& +\int_{0}^{a} f^{\prime \prime}(K)\left(K-\bar{S}^{c}\right)_{+} \mathrm{d} K+\int_{a}^{\infty} f^{\prime \prime}(K)\left(\bar{S}^{c}-K\right)_{+} \mathrm{d} K .
\end{aligned}
$$


Choosing the function $f(S)$ defined in (12), setting $a=\mathrm{E}[S]$ and taking into account that $\mathrm{E}\left[\bar{S}^{c}\right]=\mathrm{E}[S]$, we find that $\operatorname{Var}\left[\bar{S}^{c}\right]$ can be expressed as follows in terms of comonotonic index option prices:

$$
\operatorname{Var}\left[\bar{S}^{c}\right]=2 \mathrm{e}^{r T}\left(\int_{0}^{\mathrm{E}[S]} \bar{P}^{c}[K] \mathrm{d} K+\int_{\mathrm{E}[S]}^{+\infty} \bar{C}^{c}[K] \mathrm{d} K\right) .
$$

Inspired by the approximation (19) for the variance of the index price $S$, which is a linear combination of observed index option prices, we propose to approximate the variance of the comonotonic index price $\bar{S}^{c}$ by the following linear combination of comonotonic index option prices:

$$
\operatorname{Var}\left[\bar{S}^{c}\right] \approx 2 \mathrm{e}^{r T} \sum_{i=-l}^{h} \Delta K_{i} \bar{Q}^{c}\left[K_{i}\right]-\left(\mathrm{E}[S]-K_{0}\right)^{2},
$$

where as before, the $K_{i}$ are the traded index strikes, and where $\bar{Q}^{c}\left[K_{i}\right]$ is defined by

$$
\bar{Q}^{c}\left[K_{i}\right]=\left\{\begin{array}{cl}
\bar{P}^{c}\left[K_{i}\right], & \text { if } K_{i}<K_{0}, \\
\frac{\bar{C}^{c}\left[K_{i}\right]+\bar{P}^{c}\left[K_{i}\right]}{2}, & \text { if } K_{i}=K_{0}, \\
\hline \bar{C}^{c}\left[K_{i}\right], & \text { if } K_{i}>K_{0} .
\end{array}\right.
$$

Taking into account the expressions (31) and (36), we can conclude that the comonotonic index option prices $\bar{C}^{c}\left[K_{i}\right]$ and $\bar{P}^{c}\left[K_{i}\right]$, and hence also the approximation (39) for $\operatorname{Var}\left[S^{c}\right]$, can be determined in a rather straightforward way from observed stock option price data.

\section{Measuring the degree of herd behavior in stock markets}

\subsection{The implied herd behavior index}

For any of the traded strikes $K$, the index call option prices $C[K]$ can be observed in the market. From (31), it follows that for each of these strikes, we can also determine the corresponding comonotonic index call option prices $\bar{C}^{c}[K]$ from the prices of the traded European stock options. In Chen et al. (2008) it is proven that the following inequalities hold:

$$
C[K] \leq C^{c}[K] \leq \bar{C}^{c}[K] .
$$

Moreover, they prove that, given the observed call option prices of the different stocks in the market, $\bar{C}^{c}[K]$ is the price of the cheapest super-replicating strategy for the basket option $C[K]$ in a broad class of admissable investment strategies. Similar results hold for the put option case, which is considered in Linders et al. (2011). In particular, they prove that

$$
P[K] \leq P^{c}[K] \leq \bar{P}^{c}[K] .
$$

In practice, stock prices will never behave comonotonic so that the upper bounds $\bar{C}^{c}[K]$ and $\bar{P}^{c}[K]$ in (41) and (42) will never be reached. Laurence (2007) introduced 
the terminology 'comonotonicity gap' to indicate the difference between the comonotonic index option price $\bar{C}^{c}[K]$, resp. $\bar{P}^{c}[K]$, and the observed true market price $C[K]$, resp. $P[K]$, for an appropriate choice of the traded strike $K$. Obviously, this comonotonicity gap will vary over time.

In order to be able to investigate the 'variation in degree of herd behavior', hereafter we will introduce the 'Herd Behavior Index', which gives an indication of the degree of future co-movement of stock prices as implied by today's option prices. A consistent daily (or more frequent) recording of this index will reveal information about the market perception on the degree of future herd behavior and more important, about the evolution of this perception over time.

Taking into account Theorem 1, one could define the Herd Behavior Index as the proportion $\frac{\operatorname{Var}[S]}{\operatorname{Var}\left[S^{c}\right]}$. This index uses $\operatorname{Var}[S]$ to represent the real market situation and compares it with $\operatorname{Var}\left[S^{c}\right]$, which corresponds with the extreme case of comonotonicity or perfect herd behavior. The proportion takes values in the interval $[0,1]$. It equals 1 if, and only if, the market is comonotonic. In general, neither $\operatorname{Var}[S]$ nor $\operatorname{Var}\left[S^{c}\right]$ are observable. Therefore, we propose to replace $\operatorname{Var}[S]$ by its approximation (19), which is a linear combination of observed index option prices. It seems then natural to replace $\operatorname{Var}\left[S^{c}\right]$ by the approximation (39) for $\operatorname{Var}\left[\bar{S}^{c}\right]$, which is the corresponding linear combination of the comonotonic index option prices. These considerations lead to the following definition of the Herd Behavior Index.

Definition 1 Consider the random vector $\underline{X}$ representing the stock prices $X_{i}, i=1,2, \ldots, n$ at time T. The T-year implied Herd Behavior Index, notation HIX[T], is defined as

$$
H I X[T]=\frac{2 e^{r T} \sum_{i=-l}^{h} \Delta K_{i} Q\left[K_{i}\right]-\left(E[S]-K_{0}\right)^{2}}{2 e^{r T} \sum_{i=-l}^{h} \Delta K_{i} \bar{Q}^{c}\left[K_{i}\right]-\left(E[S]-K_{0}\right)^{2}} .
$$

HIX $[T]$ is a $T$ - year implied measure for the degree of herd behavior, which is calculated by comparing a weighted sum of traded stock option prices by the corresponding weighted sum of comonotonic option prices. In order to calculate HIX[T], we need the values of $Q\left[K_{i}\right]$ and $\bar{Q}^{c}\left[K_{i}\right], i=-l, \ldots, h$ as input. The $Q\left[K_{i}\right]$ follow immediately from the observed index option prices, see $(20)$. The $\bar{Q}^{c}\left[K_{i}\right]$ follow from the observed stock option prices, see (31), (36) and (40). No distributional assumptions have to be made, and in this sense, HIX $[T]$ is a model-free measure for the implied degree of herd behavior in the market. In general, there will be no options available that expire exactly at time $T$. In this case, HIX $[T]$ is calculated by means of an appropriate linear inter- or extrapolation, see Section 5.

We remark that $\operatorname{HIX}[T]$ is a generalization of the comonotonicity ratio $C[K] / \bar{C}^{c}[K]$, which is considered in Laurence (2007). Whereas the comonotonicity ratio compares a single traded index option with its comonotonic counterpart, in the calculation of the HIX all traded strikes are involved, leading to a more robust measure for the degree of herd behavior. The relation between the comonotonicity ratio and the newly introduced herd behavior index is further explored in Linders et al. (2011). 


\subsection{Implied herd behavior and correlation}

Market practicioners are well aware of the risk related to a market with strong positive dependencies between the stock prices $X_{1}, X_{2}, \ldots, X_{n}$. The most straightforward way to capture this systemic risk is via the pairwise correlations between the stock prices. In this section, we will show that these correlations may fail to capture the degree of herd behavior and could even give misleading signals, especially in highly volatile markets.

The variance of the market index price $S$ can be written as

$$
\sigma_{S}^{2}=\sum_{i=1}^{n} w_{i}^{2} \sigma_{X_{i}}^{2}+\sum_{i \neq j} w_{i} w_{j} \sigma_{X_{i}} \sigma_{X_{j}} \operatorname{corr}\left[X_{i}, X_{j}\right]
$$

It is very tempting to try to express herd behavior in terms of the $n(n-1)$ correlations between the different stock prices. Such an approach however only reflects the market's perception of the future correlations, not the future degree of herd behavior. High correlations are indeed a sign for a high degree of herd behavior in the market, but low correlations do not necessarily imply a low degree of dependence. Hence, correlations could give misleading signals. An explanation of this flaw is that one often considers the maximal variance of the random index price $S$ to arise when all correlations $\operatorname{corr}\left[X_{i}, X_{j}\right]$ are equal to 1 , which is however not true in general. Given the distributions $F_{X_{i}}$ of the marginals, the maximal attainable values for the correlations $\operatorname{corr}\left[X_{i}, X_{j}\right]$ are given by $\operatorname{corr}\left[F_{X_{i}}^{-1}(U), F_{X_{j}}^{-1}(U)\right]$, and the maximal variance of the comonotonic index price is equal to $\sigma_{S^{c}}^{2}$, which is given by

$$
\sigma_{S^{c}}^{2}=\sum_{i=1}^{n} w_{i}^{2} \sigma_{X_{i}}^{2}+\sum_{i \neq j} w_{i} w_{j} \sigma_{X_{i}} \sigma_{X_{j}} \operatorname{corr}\left[F_{X_{i}}^{-1}(U), F_{X_{j}}^{-1}(U)\right] .
$$

Although correlations fully determine the dependence structure for multivariate elliptical distributions, they fail to do so outside this class of distributions. The nonequivalence of comonotonicity and correlation 1 for a random couple can easily be illustrated by the couple $\left(X, X^{2}\right)$ where $X$ is a standard normal random variable. This couple is comonotonic, while $\operatorname{corr}\left[X, X^{2}\right]=0$. Embrechts et al. (1999) illustrate this failure by considering two lognormal random variables. In this case, the set of attainable correlations is a strict subset of $[-1,+1]$, which becomes smaller when one of the volatilities increases. Inspired by this example, hereafter we demonstrate that, given a very strong positive dependence structure between two future stock prices, their correlation can nevertheless be very low, which could be wrongly interpreted as a signal for a low degree of herd behavior. The HIX, however, is capable of detecting this strong dependence and correctly reflects the high degree of herd behavior.

Consider two stocks with price processes $\left\{X_{i}(t) \mid 0 \leq t \leq T\right\}, i=1$, 2. Suppose that their risk neutral dynamics are described by the following stochastic differential equations:

$$
\left\{\begin{array}{l}
\frac{\mathrm{d} X_{1}(t)}{X(t)}=r \mathrm{~d} t+\sigma_{1} \mathrm{~d} B_{1}(t) \\
\frac{\mathrm{d} X_{2}(t)}{X_{2}(t)}=r \mathrm{~d} t+\sigma_{2} \mathrm{~d} B_{2}(t)
\end{array}\right.
$$


where $\left\{B_{1}(t) \mid t \geq 0\right\}$ and $\left\{B_{2}(t) \mid t \geq 0\right\}$ are non-independent standard Brownian motion processes. The instantaneous correlation between these processes (under both the physical measure and the risk-neutral meausure) is given by $\rho$. The random variables $X_{1}=X_{1}(T)$ and $X_{2}=X_{2}(T)$ are both lognormal distributed with expected values and variances given by

$$
\mathrm{E}\left[X_{i}\right]=\mathrm{e}^{r T} \text { and } \sigma_{X_{i}}^{2}=X_{i}(0)^{2} \mathrm{e}^{2 r T}\left(\mathrm{e}^{\sigma_{i}^{2} T}-1\right), \quad i=1,2 .
$$

The correlation between $X_{1}$ and $X_{2}$ is equal to

$$
\operatorname{corr}\left[X_{1}, X_{2}\right]=\frac{\mathrm{e}^{\rho \sigma_{1} \sigma_{2} T}-1}{\sqrt{\mathrm{e}^{\sigma_{1}^{2} T}-1} \sqrt{\mathrm{e}^{\sigma_{2}^{2} T}-1}} .
$$

As the distribution of $\left(X_{1}, X_{2}\right)$ is completely specified, we define the HIX by

$$
\operatorname{HIX}[T]=\frac{\operatorname{Var}[S]}{\operatorname{Var}\left[S^{c}\right]}=\frac{\sigma_{X_{1}}^{2}+\sigma_{X_{2}}^{2}+2 \operatorname{corr}\left[X_{1}, X_{2}\right] \sigma_{X_{1}} \sigma_{X_{2}}}{\sigma_{X_{1}}^{2}+\sigma_{X_{2}}^{2}+2 \operatorname{corr}\left[F_{X_{1}}^{-1}(U), F_{X_{2}}^{-1}(U)\right] \sigma_{X_{1}} \sigma_{X_{2}}},
$$

where $\operatorname{corr}\left[F_{X_{1}}^{-1}(U), F_{X_{2}}^{-1}(U)\right]$ is the maximal correlation between $X_{1}$ and $X_{2}$ :

$$
\operatorname{corr}\left[F_{X_{1}}^{-1}(U), F_{X_{2}}^{-1}(U)\right]=\frac{\mathrm{e}^{\sigma_{1} \sigma_{2} T}-1}{\sqrt{\mathrm{e}^{\sigma_{1}^{2} T}-1} \sqrt{\mathrm{e}^{\sigma_{2}^{2} T}-1}} .
$$

In the remainder of this example, we choose the following numerical values for the parameters involved: $r=0.03, T=1, \sigma_{1}=0.2$ and $\rho=0.95$. The choice of the instantaneous correlation implies that $\operatorname{corr}\left[B_{1}(t), B_{2}(t)\right]=0.95$, and hence also that

$$
\operatorname{corr}\left[\ln X_{1}(t), \ln X_{2}(t)\right]=0.95, \quad 0 \leq t \leq T .
$$

Taking into account that for a bivariate normal random pair, comonotonicity is equivalent with a correlation of 1 , we find that $\left(\ln X_{1}(t), \ln X_{2}(t)\right)$, and thus also $\left(X_{1}(t), X_{2}(t)\right)$, is close to being comonotonic. This means that at any time $t$, the stock prices $X_{1}(t)$ and $X_{2}(t)$ are strongly positive dependent.

Figure 1 shows the herd behavior index HIX[1] and correlation $\operatorname{corr}\left[X_{1}(1), X_{2}(1)\right]$ for different values of $\sigma_{2}$. When the volatility $\sigma_{2}$ increases, both the HIX and the correlation are changing, but this behavior is much more pronounced for the correlation. In fact, HIX[1] tends to 1 whereas $\operatorname{corr}\left[X_{1}(1), X_{2}(1)\right]$ goes to 0 .

Intuitively, we may explain this limiting behavior as follows: in case $X_{2}$ has a much larger variance than $X_{1}$, we have that $X_{1}$ almost behaves as a constant value, compared to $X_{2}$. But the correlation between a random variable and a constant is 0 , while at the same time, they are comonotonic.

We can conclude that in markets with some highly volatile stocks, correlation may fail to capture the underlying dependence in the right way. This dysfunctioning of correlation is caused by the non-linear relationship between the random variables and becomes clearer when $\sigma_{2}>1$. Based on usual market practice, volatilities larger than 1 seem to be unrealistic, unless we are dealing with a very distressed market. But it is exactly in such a situation that we might need an accurate estimate of the degree of implied herd behavior and systemic risk. At such a crucial moment, correlations may give a completely wrong indication, whereas the HIX is capable of providing us with the correct information. 


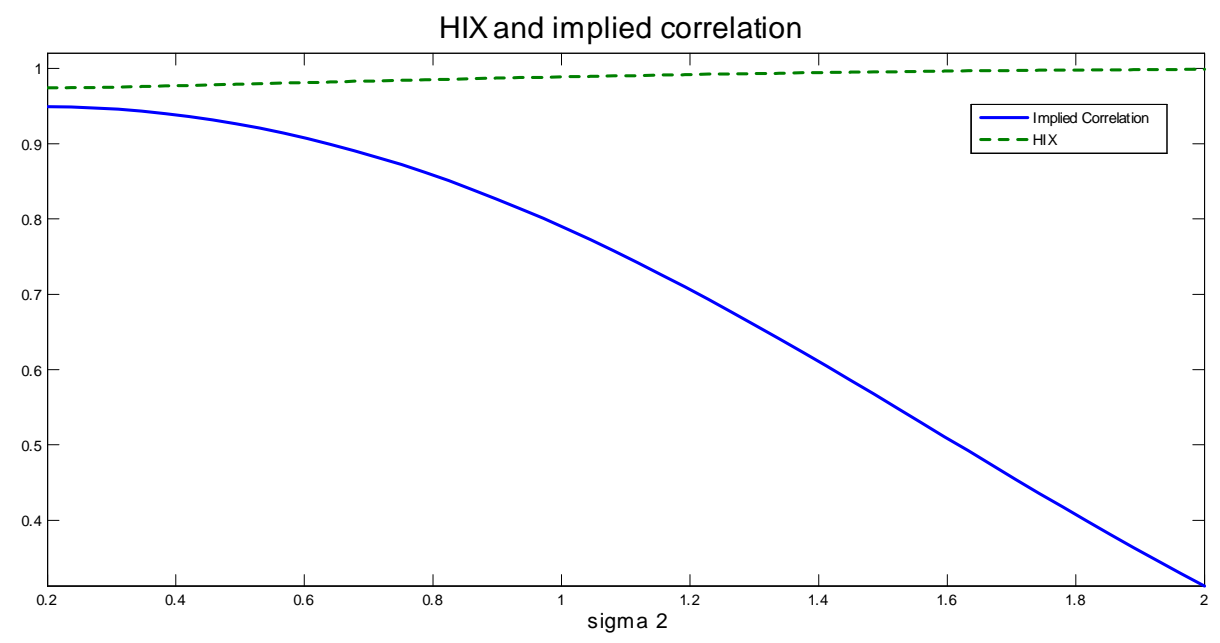

Figure 1: $\operatorname{Corr}\left[X_{1}(1), X_{2}(1)\right]$ and HIX[1] for different values of $\sigma_{2}$.

\subsection{Measuring implied herd behavior via the VIX methodology}

The key quantities in the definition of the HIX are the variances of the index price and the comonotonic index price. In this subsection, we explain how the approach for calculating the HIX out of observed options prices can also be used for determining a herd behavior index based on the VIX methodology. For completeness, we first shortly describe the VIX and the related return variance swap contracts. For a more detailed discussion on the VIX, the reader is referred to Carr and Wu (2006) and Chicago Board Options Exchange (2009).

In 1993, the Chicago Board Options Exchange (CBOE) introduced the CBOE Volatility Index (ticker symbol VIX), which since then has become the industry benchmark for market volatility. In 2003, CBOE launched a renewed version of the VIX Index. This new VIX is calculated based on S\&P 500 index (SPX) option prices. VIX can be interpreted as a quote on the expected market volatility over the next 30 calendar days. To be more precise, VIX squared is an approximation of the 30-day return variance swap rate on the SPX.

30-day return variance swaps on the S\&P 500 index are sold over-the-counter. Let us consider such a contract that is initiated today, at time 0 . At maturity, i.e. at time $T=30 / 365$, one leg of the swap pays the buyer the (annualized) realized variance $\operatorname{RV}[T]$ of the logprice changes of the index:

$$
\mathrm{RV}[T]=\frac{1}{T} \sum_{j=1}^{30}\left(\ln S\left(\frac{j}{365}\right)-\ln S\left(\frac{j-1}{365}\right)\right)^{2} .
$$

The other leg pays the seller the fixed amount $\mathrm{SR}[T]$ at time $T$, which is the swap rate that is agreed upon at the deal's inception (time 0), and which is determined such that the risk-neutral price of the pay-off ( $\mathrm{RV}[T]-\mathrm{SR}[T])$ at time $T$ is zero at inception, hence

$$
\mathrm{SR}[T]=\mathrm{E}[\mathrm{RV}[T]] .
$$


The buyer of the variance swap is long volatility. A variance swap contract allows one to speculate on the future realized variance or hedge against risks associated with the magnitude of movement of the index.

Under a fairly general setting for the $\mathbb{Q}$ - dynamics of the assets involved, and also assuming a continuously (instead of daily) sampled variance over the lifetime of the contract, Carr and $\mathrm{Wu}(2006)$ prove that the realized variance is given by

$$
\mathrm{RV}[T]=\frac{2}{T}\left(\frac{S}{\mathrm{E}[S]}-1-\ln \left(\frac{S}{\mathrm{E}[S]}\right)\right)+A[T]+B[T],
$$

where $A[T]$ is the pay-off at time $T$ of a dynamic trading strategy in futures for which $\mathrm{E}[A[T]]=0$, and $B[T]$ is a higher order term induced by the jumps in the index price dynamics. Applying (9) with $f(S)=\ln \left(\frac{S}{\mathrm{E}[S]}\right)$ and $a=\mathrm{E}[S]$, and substituting this expression for $f(S)$ in (48) leads to

$$
\mathrm{RV}[T]=\frac{2}{T}\left(\int_{0}^{\mathrm{E}[S]} \frac{(K-S)_{+}}{K^{2}} d K+\int_{\mathrm{E}[S]}^{+\infty} \frac{(S-K)_{+}}{K^{2}} d K\right)+A[T]+B[T] .
$$

This expression shows that, up to the futures component $A[T]$ and the higher order jump component $B[T]$, the return variance can be replicated by the pay-off from a static position in a continuum of European options on the index. Taking expectations with respect to $\mathbb{Q}$, we obtain

$$
\mathrm{SR}[T]=\frac{2}{T} \mathrm{e}^{r T}\left(\int_{0}^{\mathrm{E}[S]} \frac{P[K]}{K^{2}} \mathrm{~d} K+\int_{\mathrm{E}[S]}^{+\infty} \frac{C[K]}{K^{2}} \mathrm{~d} K\right)+\mathrm{E}[B[T]],
$$

which shows that the swap rate is equal to the sum of a weighted average of index option prices across all strikes and a higher order term. Ignoring the higher order term and further approximating the remaining integrals in a similar way as the one that led to the approximation (19) for $\operatorname{Var}[S]$, we find the following approximate expression for the variance swap rate $\mathrm{SR}[T]$ in terms of observed option prices:

$$
\operatorname{SR}[T] \approx \sigma^{2}[T]=: \frac{2}{T} \mathrm{e}^{r T} \sum_{i=-l}^{h} \frac{\Delta K_{i}}{K_{i}^{2}} Q\left[K_{i}\right]-\frac{1}{T}\left(\frac{\mathrm{E}[S]}{K_{0}}-1\right)^{2},
$$

where $\Delta K_{i}$ and $Q\left[K_{i}\right]$ are defined as before. The approximation $\sigma^{2}[T]$ for $\operatorname{SR}[T]$ is crucial in the VIX calculation. Choosing $T$ equal to 30 calendar days, interpreting all notations above in terms of the S\&P 500 index and assuming that there are options available that expire in exactly 30 days, the VIX is defined as VIX $[T]=100 \times \sigma[T]$. Usually however, there will be no options available that expire exactly in 30 calendar days. The $T=30$ calendar days VIX is then calculated by using the appropriate linear inter- or extrapolation, see Section 5.

The VIX is a volatility index which is considered by the market as an indicator for market stress. Based on the VIX methodology, CBOE also calculates volatility indices for other markets, including the CBOE DJ Volatility Index (VXD). 
We now define the comonotonic version of $\sigma^{2}[T]$ by replacing the index option prices $P[K]$ and $C[K]$ in (50) by the corresponding comonotonic index option prices $\bar{P}^{c}[K]$ and $\bar{C}^{c}[K]:$

$$
\left(\bar{\sigma}^{c}\right)^{2}[T]=\frac{2}{T} \sum_{i=-l}^{h} \frac{\Delta K_{i}}{K_{i}^{2}} \mathrm{e}^{r T} \bar{Q}^{c}\left[K_{i}\right]-\frac{1}{T}\left(\frac{\mathrm{E}[S]}{K_{0}}-1\right)^{2} .
$$

In case there are options available that expire in exactly $T=30$ days, the comonotonic VIX is then defined as VIX ${ }^{c}[T]=100 \times \bar{\sigma}^{c}[T]$. Inspired by the methodology described in Section 4.1, Dhaene et al. (2011) introduce the $T$ - year implied Comonotonicity Index. We slightly adapt their definition, and define the CIX as the ratio of the (approximated) swap rate to the comonotonic swap rate:

$$
\mathrm{CIX}[T]=\frac{\sigma^{2}[T]}{\left(\bar{\sigma}^{c}\right)^{2}[T]},
$$

provided options that expire at time $T$ are traded in the market. The CIX is an alternative measure for herd behavior and systemic risk. It can be interpreted as the ratio of VIX squared (which is based on observed index option prices $Q\left[K_{i}\right]$ ) to the comonotonic VIX squared (which is based on comonotonic index option prices $\bar{Q}^{c}\left[K_{i}\right]$ ). We refer to Dhaene et al. (2011) for a more extensive study of the systemic risk indicator based on the VIX.

\section{$5 \quad$ Numerical issues}

Suppose that for a particular financial market, we want to calculate today's value of the $T$ - year HIX. In this section, we consider several numerical issues related to the calculation of this value.

Let us first assume that stock options as well as index options with maturity $T$ years (e.g. $T=\frac{30}{365}$ ) are traded. In practice, we will not observe the theoretical index call option price $C[K]$ for each traded strike $K$. Instead, we will observe a bid price $C^{b i d}[K]$ and a larger ask price $C^{a s k}[K]$. In order to cope with this bid/ask spread, we propose to use midquote prices as an approximation for the theoretical option prices:

$$
C[K] \approx \frac{C^{b i d}[K]+C^{a s k}[K]}{2} .
$$

Similar conventions are made for put options on the index as well as for call and put options on the individual stocks. Hereafter, we will always refer to midquote prices when considering option prices.

The HIX formula (43) contains the forward index price $\mathrm{E}[S]$. In line with the VIX methodology, we propose to calculate $\mathrm{E}[S]$ based on the put-call parity (13) for the pair of index put and call options with prices that are closest to each other. Hence,

$$
\mathrm{E}[S]=\mathrm{e}^{r T}\left(C\left[K_{i}^{*}\right]-P\left[K_{i}^{*}\right]\right)+K_{i}^{*},
$$

where

$$
K_{i}^{*}=\arg \min _{K \in\left\{K_{-l}, \ldots, K_{h}\right\}}|C[K]-P[K]| .
$$


We have assumed that for each stock $i$, the maximal value $K_{i, m_{i}+1}$ of $X_{i}$ is finite. As mentioned before, the optimal choice of these $K_{i, m_{i}+1}$ is considered in Chen et al. (2008). The observed prices $C_{i}\left[K_{i, j}\right]$ or $P_{i}\left[K_{i, j}\right]$ of the options written on the individual stocks are used to construct the empirical distribution function $\bar{F}_{X_{i}}$, by first introducing the piecewise linear functions $\bar{C}_{i}[K]$ or $\bar{P}_{i}[K]$ and then applying (5) or (8). This leads to the expressions (4) or (7) for $\bar{F}_{X_{i}}$. In this procedure, it is implicitely assumed that the option prices $C_{i}[0], P_{i}[0], C_{i}\left[K_{i, m_{i}+1}\right]$ and $P_{i}\left[K_{i, m_{i}+1}\right]$ are given. Obviously, we have that

$$
C_{i}\left[K_{i, m_{i}+1}\right]=P_{i}[0]=0 .
$$

Furthermore, the theoretical option prices $C_{i}[0]$ and $P_{i}\left[K_{i, m_{i}+1}\right]$ are given by

$$
C_{i}[0]=\mathrm{e}^{-r T} \mathrm{E}\left[X_{i}\right]
$$

and

$$
P_{i}\left[K_{i, m_{i}+1}\right]=\mathrm{e}^{-r T}\left(K_{i, m_{i}+1}-\mathrm{E}\left[X_{i}\right]\right) .
$$

Inspired by the above-mentioned approach to determine $\mathrm{E}[S]$, we propose to calculate $\mathrm{E}\left[X_{i}\right]$ as follows:

$$
\mathrm{E}\left[X_{i}\right]=\mathrm{e}^{r T}\left(C_{i}\left[K_{i}^{*}\right]-P\left[K_{i}^{*}\right]\right)+K_{i}^{*}
$$

with

$$
K_{i}^{*}=\arg \min _{K \in\left\{K_{i, 1}, \ldots K_{i, m_{i}}\right\}}\left|C_{i}[K]-P[K]\right| .
$$

Plugging these values of the $\mathrm{E}\left[X_{i}\right]$ in (58) and (59) leads to the quotes for the call options with strike 0 and put options with strike $K_{i, m_{i}+1}$ that we will use in the calculations.

In order to make the HIX sufficiently stable, we only use stock options which have a bid price which is strictly larger than zero and a volume which is strictly larger than 20 for determining the risk-neutral distributions $\bar{F}_{X_{i}}$. Notice that it may happen that for one or more of the underlying stocks $i$, the only option prices available are the ones with strikes 0 and $K_{m_{i}+1}$. This situation may occur if the market is illiquid or because there are no options issued on that particular stock. In this case, the HIX can still be determined according to the methodology presented above. This situation is considered in more detail in Linders et al. (2011).

Due to price irregularities, it may happen that the piecewise linear function $\bar{C}_{i}[K]$ or $\bar{P}_{i}[K]$ is not convex, leading to a function $\bar{F}_{X_{i}}$ that is not increasing and hence, not a proper cumulative distribution function. In order to circumvent this problem, we propose to work with the function $\widetilde{F}_{X_{i}}$ instead of $\bar{F}_{X_{i}}$, which is defined as follows:

$$
\widetilde{F}_{X_{i}}\left(K_{i, j}\right)=\min \left\{\bar{F}_{X_{i}}\left(K_{i, j}\right), \widetilde{F}_{X_{i}}\left(K_{i, j+1}\right)\right\}, \quad j=0,1, \ldots, m_{i}
$$

with initial value

$$
\widetilde{F}_{X_{i}}\left(K_{i, m_{i}+1}\right)=1
$$

Until here, we assumed that all options on the index as well as on the individual stocks are of the European type. In the next section, we will apply our methodology 
to the Dow Jones Industrial Average (DJ) index. In this case, the index options are of European type, whereas the individual stock options are of American type, where the holder has the right to exercise the option at any time up to and including the maturity date. In general, the price of an American option is an upper bound for the corresponding European option. Therefore, we will continue to use the methodology described above, but we replace the (non-observed) European option prices $C_{i}\left[K_{i, j}\right]$ and $P_{i}\left[K_{i, j}\right]$ that appear in the expressions (31) and (36) for $\bar{C}^{c}[K]$ and $\bar{P}^{c}[K]$ by the corresponding (observed) American option prices. As the option prices $C_{i}\left[K_{i, j}\right]$ and $P_{i}\left[K_{i, j}\right]$ only appear in the denominator of the HIX and the CIX, this approximation will lead to somewhat smaller values for the respective indices.

Suppose now that we want to calculate the $T$ - year HIX on a regular (e.g. hourly or daily) basis. As an example, hereafter we set $T$ equal to 30 calendar days, hence $T=\frac{30}{365}$. Usually, no options will expire in exactly 30 calendar days. Let us denote the first available maturity date by $T_{1}$ and the next one by $T_{2}$. Options which mature at time $T_{1}$ are called near-term options, the ones which mature at time $T_{2}$ are called next-term options. Inspired by the methodology used for calculating the VIX, the Herd Behavior Index with maturity $T$ is now calculated as a weighted average of the near-term and the next-term Herd Behavior Index:

$$
\operatorname{HIX}[T]=\operatorname{HIX}\left[T_{1}\right] \times\left[\frac{T_{2}-T}{T_{2}-T_{1}}\right]+\operatorname{HIX}\left[T_{2}\right] \times\left[\frac{T-T_{1}}{T_{2}-T_{1}}\right] .
$$

In case that for each month only a single expiration date is available in the market (e.g. the opening of the third Friday of the month), we have that $T_{1} \leq T \leq T_{2}$ and formula (64) for $\operatorname{HIX}[T]$ is an interpolation of $\operatorname{HIX}\left[T_{1}\right]$ and $\operatorname{HIX}\left[T_{2}\right]$. Notice that the risk-free interest rate used for calculating $\operatorname{HIX}\left[T_{i}\right]$ is set equal to the risk free interest rate to expiration $T_{i}, i=1,2$. This implies that different risk-free interest rates may be used for near- and next-term options. In order to avoid possible price irregularities near to expiration, we 'roll' the HIX to the second and the third contract months in case the near-term options have less than a week to expiration. After such a roll, we will encounter a situation where $T<T_{1}<T_{2}$, with $T_{1}$ and $T_{2}$ now standing for the second and third expiration dates, respectively. In this case, formula (64) for $\operatorname{HIX}[T]$ is an extrapolation of $\operatorname{HIX}\left[T_{1}\right]$ and $\operatorname{HIX}\left[T_{2}\right]$.

It may happen that the near- and the next-term maturities $T_{i, 1}$ and $T_{i, 2}$ of options on stock $i$ differ from the near- and the next-term maturities $T_{1}$ and $T_{2}$ of the stock index. In the numerical illustration in the next section, these differences $\left|T_{k}-T_{i, k}\right|, k=1,2$, are small, i.e. typically only a few days. Therefore, when this situation occurs, we will approximate the (non-observed) required option prices $C_{i}\left[K_{i, j}, T_{k}\right]$ by the observed quotes $C_{i}\left[K_{i, j}, T_{i, k}\right]$.

In the next section, we will also calculate historical values of the CIX, which was defined in (53), according to the same methodology as the one presented above for the HIX. In particular, we will calculate the CIX with maturity $T$ as a weighted average of the near-term and the next-term indices:

$$
\operatorname{CIX}[T]=\operatorname{CIX}\left[T_{1}\right] \times\left[\frac{T_{2}-T}{T_{2}-T_{1}}\right]+\operatorname{CIX}\left[T_{2}\right] \times\left[\frac{T-T_{1}}{T_{2}-T_{1}}\right] .
$$


Notice that this way of determining a herd behavior index based on the VIX volatility index is somewhat different from the one proposed in Dhaene et al. (2011), where the linear inter- or extrapolation is not performed at the level of the CIX, but at the level of the numerator and the denominator in (53) separately. Based on the VIX inter- or extrapolation formula

$$
\operatorname{VIX}[T]=100 \sqrt{\frac{1}{T}\left\{T_{1} \sigma^{2}\left[T_{1}\right] \times\left[\frac{T_{2}-T}{T_{2}-T_{1}}\right]+T_{2} \sigma^{2}\left[T_{2}\right] \times\left[\frac{T-T_{1}}{T_{2}-T_{1}}\right]\right\}},
$$

these authors introduce the following comonotonic upper bound for the VIX:

$$
\operatorname{VIX}^{c}[T]=100 \sqrt{\frac{1}{T}\left\{T_{1}\left(\bar{\sigma}^{c}\right)^{2}\left[T_{1}\right] \times\left[\frac{T_{2}-T}{T_{2}-T_{1}}\right]+T_{2}\left(\bar{\sigma}^{c}\right)^{2}\left[T_{2}\right] \times\left[\frac{T-T_{1}}{T_{2}-T_{1}}\right]\right\}}
$$

and propose to measure the herd behavior index by the ratio $\frac{\operatorname{VIX}[T]}{\operatorname{VIX}^{c}[T]}$. This index has a somewhat more attractive look compared to the CIX defined above, but the way how it is constructed out of near and next term options is somewhat less appropriate from a theoretical point of view.

\section{Numerical illustration: the HIX for the Dow Jones}

The Dow Jones. The Dow Jones Industrial Average (DJ), established 1896, is a priceweighted index composed of the 30 largest, most liquid NYSE and NASDAQ listed stocks. Besides being the oldest continuing market index, it is probably also the world's best known stock market index to individual investors. Options with the DJ index as underlying are called DJX options. These (European-type) options were introduced in 1997 and have become some of the most popular index options worldwide. DJX index options are based on 1/100th of the current value of the DJ. Therefore, hereafter $S(t)$ has to be interpreted as 1/100th of the value of the DJ at time $t$. There are also (American-type) options traded on each individual component of the Dow Jones. Roughly speaking, for each stock there are around 10 traded strikes. The expiration date of the DJX options is always equal to the saturday, following the third friday of the month.

Herd behavior over time. In this section, we investigate the degree of herd behavior of the 30 stocks in the DJ by introducing the implied DJ-HIX, which is an implied short term measure for systemic risk in the DJ market. In particular, we set $T$ equal to 30 calendar days. We calculate the historical DJ-HIX values on a daily basis for the period January 2006 - October 2009. For each trading day, we use the closing bid and ask prices of the options involved. It may happen (rarely) that the near- and the next-term maturities $T_{i, 1}$ and $T_{i, 2}$ of options on stock $i$ differ from $T_{1}$ and $T_{2}$. When this situation occurs, we will approximate the required option prices $C_{i}\left[K_{i, j}, T\right]$ by $C_{i}\left[K_{i, j}, T_{i, 1}\right]$.

The first graph of Figure 2 shows the historical DJ index price levels from January 2006 
Dow Jones Industrial Average
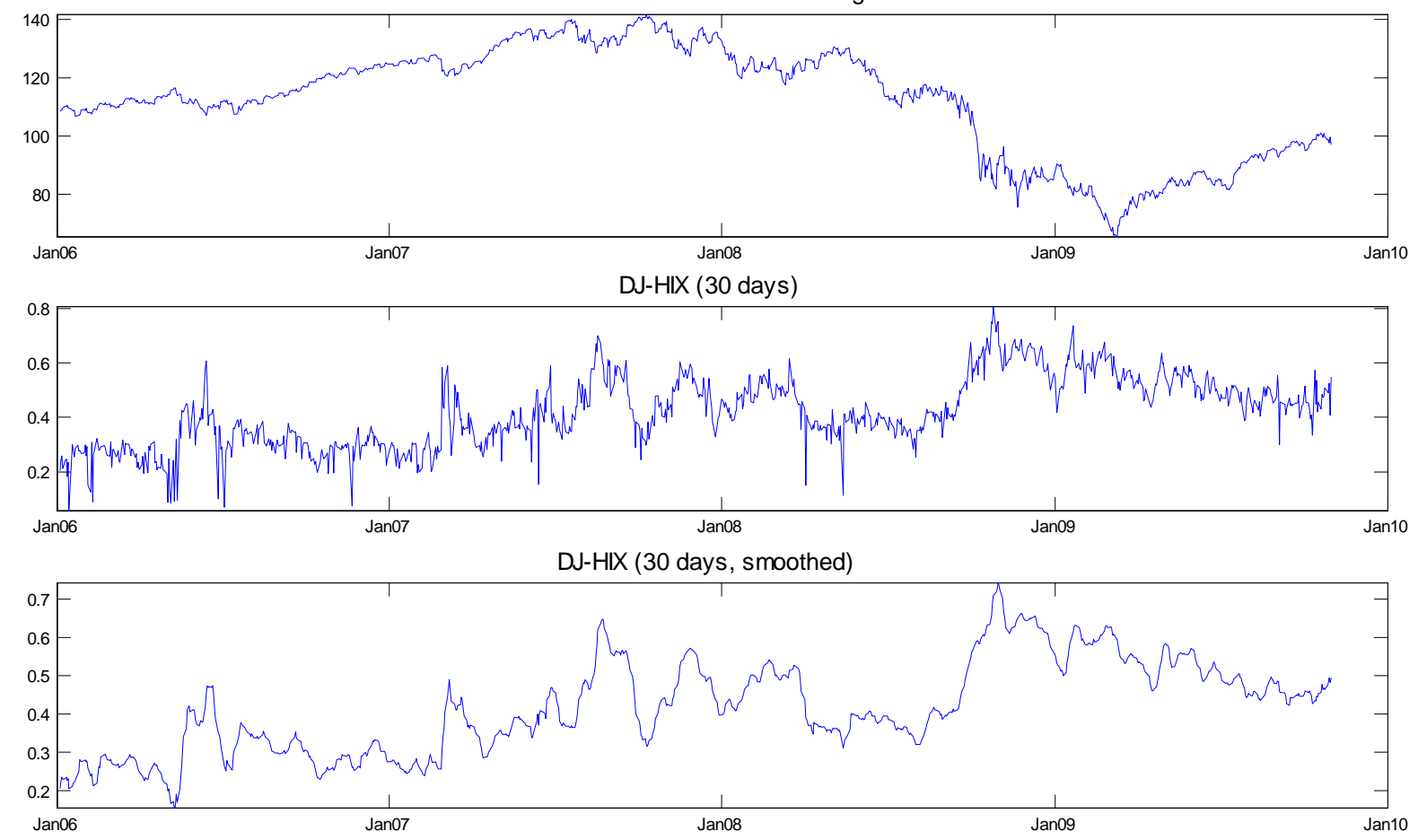

Figure 2: The Dow Jones Industrial Average, DJ-HIX(30 days) and DJ-HIX (30 days, smoothed).

until October 2009. Taking into account (43) and (64), we determine the degree of herd behavior for any day in the observation period by the DJ-HIX for $T=30$ days. These values are presented in the second graph of Figure 2. A smoothed version of these DJ-HIX values, based on the average quote over the last seven trading days, is shown in the third graph.

From Figure 2, we conclude that the DJ-HIX fluctuates substantially over time. Loosely speaking, between January 2006 and January 2007 the degree of herd behavior is relatively low, during January 2007-October 2008 it is at an intermediate level, while in the remaining part of the observation period (October 2008-October 2009), it is at a relatively high level. The DJ-HIX frequently spikes upward. From early 2007 until mid 2008 a few relatively high peaks are observed, indicating that the market was showing already signs of stress before the worldwide financial crisis towards the end of 2008. Around the middle of 2008, the market seems to calm down, but in October 2008 the DJ-HIX increases drastically and reaches its highest level of around 0.75 on October, 24. In 2009, the DJ-HIX relaxes, but only at a very slow rate, and hence, remains relatively high during the whole year. 
Herd behavior as a component of stock market fear. An increased DJ-HIX is a sign that option traders in the market believe in a stronger co-movement of the different stock prices over the next 30 days. The degree of implied herd behavior may reach a high level due to panic and a strong belief that stock prices will go down all together, inducing that also the market index will decrease rapidly. In principle, the HIX may also reach high levels due to positive financial information and a believe that in the near future all stock prices will move up together. From the observed data we find that the HIX shows a tendency to increase when the market index returns decrease. In this respect, the HIX may be viewed as a fear or stress indicator.

In Figure 3, we compare the (smoothed) DJ-HIX and the DJ Volatility Index (VXD). The latter index is a volatility barometer for the DJ, calculated according to the VIX methodology. Both the HIX and the VIX may explain part of the total market stress or market fear, see Dhaene et al. (2011). The HIX measures the implied co-movement of the components of the index, whereas the VIX-based Volatility Index measures the implied volatility of the index. Notice that an increased index volatility may be caused by increased volatilities of the components and/or by an increased degree of herd behavior. In Figure 3 we observe a tendency of the HIX to increase when the market volatility increases. The peaks in the graphs of the DJ-HIX and the DJ implied volatility index are reflecting periods of increased market stress. Notice that the DJ-HIX is a relative and bounded measure with maximal value equal to 1 in case of perfect co-movement, whereas the DJ volatility index is an absolute measure without upper bound. This latter observation explains why it may be more difficult to detect peaks in the DJ volatility index, especially in periods where this implied volatility is at a relatively low level.

The HIX versus the CIX. The HIX quantifies the degree of herd behavior in stock markets by comparing the real market situation with a synthetic one where there is perfect herd behavior. The HIX uses the estimate (19) of the variance of index price $S$ and the estimate (39) of the variance of the comonotonic index price $\bar{S}^{c}$ to represent these two situations. In Section 4.3, we presented the CIX as an alternative for the HIX. The CIX is based on the VIX-methodology. Essentially, it considers the VIX-squared to describe the real market situation, and compares it with the comonotonic VIX-squared. Both the (smoothed) DJ-HIX and the (smoothed) DJ-CIX for $T=30$ days are shown in Figure 4. We observe that both measures lead to an almost identical picture. An explanation for this observation follows from a Taylor expansion of the realized variance around $\mathrm{E}[S]$. Indeed, from (48) we find that

$$
\mathrm{RV}[T]=\frac{1}{T}\left(\frac{S-\mathrm{E}[S]}{\mathrm{E}[S]}\right)^{2}+\ldots
$$

The variance swap rate is then given by

$$
\mathrm{SR}[T]=\frac{1}{T} \frac{\operatorname{Var}[S]}{\mathrm{E}^{2}[S]}+\ldots
$$

The HIX is based on the ratio of $\operatorname{Var}[S]$ to $\operatorname{Var}\left[\bar{S}^{c}\right]$, whereas the CIX is based on the ratio of the swap rate $\mathrm{SR}[T]$ to the comonotonic version of the swap rate. Taking into account 
Dow Jones Industrial Average
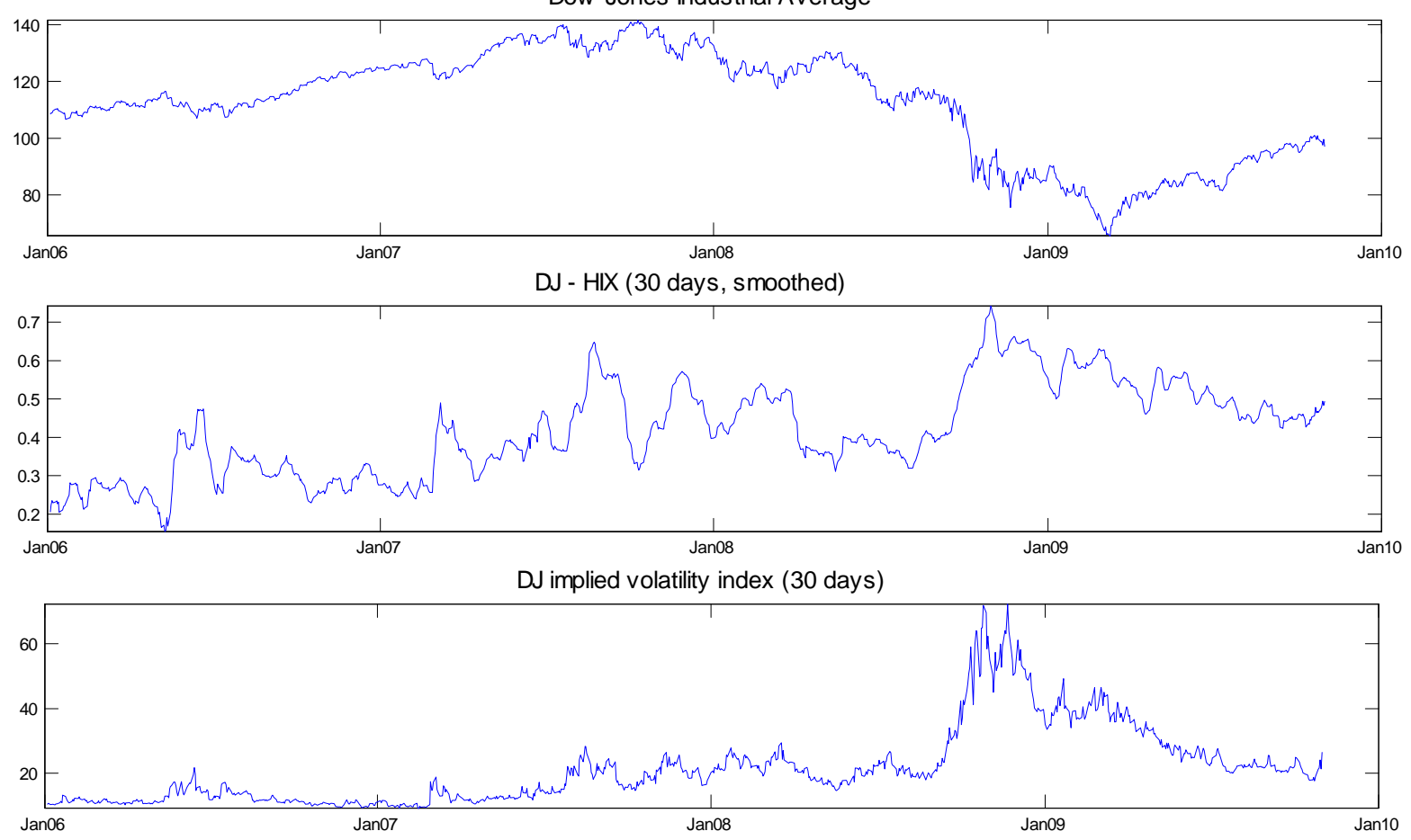

Figure 3: DJIA, DJ-HIX (30 days) and DJ-Volatility Index (30 days). 


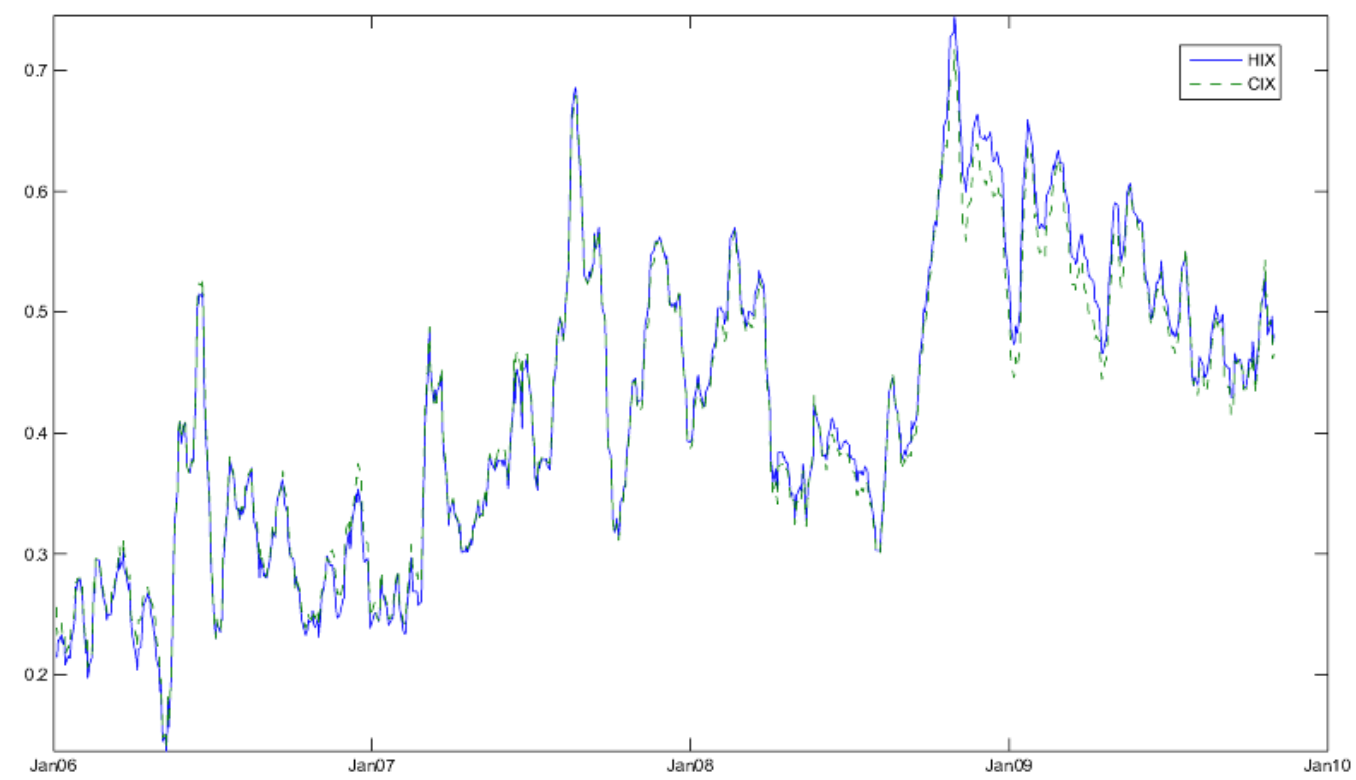

Figure 4: DJ-HIX (30 days, solid line) and DJ-CIX (30 days, dotted line).

(69), we find that HIX $[T]$ and CIX $[T]$ are equal, provided the higher order terms can be ignored.

\section{Concluding remarks}

After having experienced the late-2000s financial crisis and the related near-meltdown of the financial system, systemic risk has become in the center of the attention of stakeholders including regulators, policy makers, market supervisors, speculators and other market participants. A high level of systemic risk reflects the 'low probability, high impact event' of a market mainly driven by a single factor. Such a situation may lead to a collapse of the entire system with devastating consequences for society: the boat loses stability and may even capsize if all its passengers together run from one side to the other over and over again. Therefore, objective, forward looking and market implied estimation of systemic risk is of utmost importance. It gives market players an insight and an opportunity to take the necessary actions.

In this paper we made a modest contribution to this complicated matter by proposing a new measure for the degree of co-movement or herd behavior present in equity markets. This new measure estimates the systemic risk by comparing the currently observed market situation with the comonotonic situation under which the whole system is driven just by a single factor. More precisely, it compares an estimate of the variance of the market index 
(or basket) with an estimate of the corresponding worst-case or comonotonic variance. In line with the VIX methodology, the estimate for the variance of the market index is based on the full spectrum of current option information on the index. Although the worst-case market situation is not observed, the comonotonic variance can easily be backed out of the option prices on the constituents of the market index.

The ratio of the market-based variance and its comonotonic counterpart was baptized the Herd Behavior Index (HIX). This forward looking indicator for co-movement behavior or systemic risk is model-independent and easy to calculate. The HIX attains values between zero and 1. Today's value of the HIX expresses the market's perception of future realized herd behavior as implied by today's option prices. A level close to 1 points to a high degree of herd behavior, a lower value indicates lower degrees of co-movement. The HIX can be determined for any market index or basket with underlying traded vanilla options on the index as well as on its constituents.

Measuring the degree of co-movement with the HIX has several advantages compared to implied correlation. The HIX is able to capture all kinds of dependencies between stock prices, whereas the implied correlation is a weighted average of pairwise correlations amongst the index asset returns and hence, only focuses on linear dependencies. Furthermore, making abstraction of the approximations involved in its calculation, the HIX reaches its maximal value of 1 if and only if the underlying random variables are comonotonic. On the other hand, there is no direct link between the degree of herd behavior and the value of the implied correlation. Finally, the HIX is a model-independent estimate for future co-movement, whereas the implied correlation isn't, due to the involved Black \& Scholes implied volatilies.

We also discussed the CIX, which is an indicator for systemic risk, based on the VIX methodology. We illustrated the HIX and the CIX by determining their historical values for the Dow Jones Industrial Average. We observed that and explained why both indices are almost identical. Furthermore, we concluded that, similar to volatility indices and correlation indices, the herd behavior indices exhibit a tendency to increase when the stock prices are decreasing. The HIX/CIX could be used as a trading signal to anticipate on a bear market or to step in a correlation trade, see for example Laurence and Wang (2008) or Laurence (2007). One could monetize the gap between the numerator and denominator of the HIX/CIX by taking the appropriate position in options on one of these indices and its constituent stocks. In this way, one could take a pure exposure in, or hedge against systemic risk.

The study of applications of the HIX/CIX to financial economics problems such as hedging systemic risk is an interesting topic of future research. Further possible research topics include investigating the relationship between the HIX/CIX and the VIX-based implied volatility index, the relationship between the HIX/CIX and implied correlation. Also the performance of the HIX/CIX as a forecast for the future degree of herd behavior between assets in the underlying index has to be investigated. A somewhat related paper in this respect is Harmon et al. (2011). These authors find that the latest financial crisis and also earlier large single-day panics in stock markets were preceded by extended periods of high levels of market mimicry, measured in terms of the daily fraction of options that move in the same direction. Other empirical issues to be investigated include mean-reverting 
behavior of the HIX/CIX, clustering behavior (Are large values likely to be followed by large values?) and asymetry behavior (Have negative returns a larger impact than positive returns of the same size?), amongst others. 
Acknowledgement. Jan Dhaene, Daniël Linders and Wim Schoutens acknowledge the financial support of the Onderzoeksfonds K.U. Leuven (GOA/07: Risk Modeling and Valuation of Insurance and Financial cash Flows, with Applications to Pricing, Provisioning and Solvency and GOA/12/002/TBA: Management of Financial and Actuarial Risks: Modeling, Regulation, Incentives and Market Effects).

\section{Appendix}

\subsection{Proof of formula (19)}

The approximation (19) follows by rewriting (15) as

$$
\frac{\mathrm{e}^{-r T}}{2} \operatorname{Var}[S]=\int_{-\infty}^{K_{0}} P[K] \mathrm{d} K+\int_{K_{0}}^{+\infty} C[K] \mathrm{d} K+\int_{K_{0}}^{\mathrm{E}[S]}(P[K]-C[K]) \mathrm{d} K,
$$

see Figure 5. We split the first integral in two parts,

$$
\int_{-\infty}^{K_{0}} P[K] \mathrm{d} K=\int_{-\infty}^{K_{-l}} P[K] \mathrm{d} K+\int_{K_{-l}}^{K_{0}} P[K] \mathrm{d} K
$$

and approximate the second term by the composite trapezoidal rule

$$
\begin{aligned}
\int_{K_{-l}}^{K_{0}} P[K] \mathrm{d} K & \approx \sum_{I=-l+1}^{0}\left(K_{i}-K_{i-1}\right) \frac{P\left[K_{i-1}\right]+P\left[K_{i}\right]}{2} \\
& =\frac{K_{-l+1}-K_{-l}}{2} P\left[K_{-l}\right]+\sum_{i=-l+1}^{-1} \frac{K_{k+1}-K_{k-1}}{2} P\left[K_{i}\right]+\frac{K_{0}-K_{-1}}{2} P\left[K_{0}\right]
\end{aligned}
$$

Assuming that $P[K]$ reaches 0 in $K_{-l}-\left(K_{-l+1}-K_{-l}\right)$ we can approximate the first term by

$$
\int_{-\infty}^{K_{-l}} P[K] \mathrm{d} K \approx \frac{K_{-l+1}-K_{-l}}{2} P\left[K_{-l}\right]
$$

and thus

$$
\int_{-\infty}^{K_{0}} P[K] \mathrm{d} K \approx\left(K_{-l+1}-K_{-l}\right) P\left[K_{-l}\right]+\sum_{i=-l+1}^{-1} \frac{K_{i+1}-K_{i-1}}{2} P\left[K_{i}\right]+\frac{K_{0}-K_{-1}}{2} P\left[K_{0}\right]=: I_{1} .
$$

Analogously we find

$$
\int_{K_{0}}^{+\infty} C[K] \mathrm{d} K \approx \frac{K_{1}-K_{0}}{2} C\left[K_{0}\right]+\sum_{i=1}^{h-1} \frac{K_{i+1}-K_{i-1}}{2} C\left[K_{i}\right]+\left(K_{h}-K_{h-1}\right) C\left[K_{h}\right]=: I_{2},
$$

where we assumed that $C[K]$ reaches 0 in $K_{h}+\left(K_{h}-K_{h-1}\right)$. 


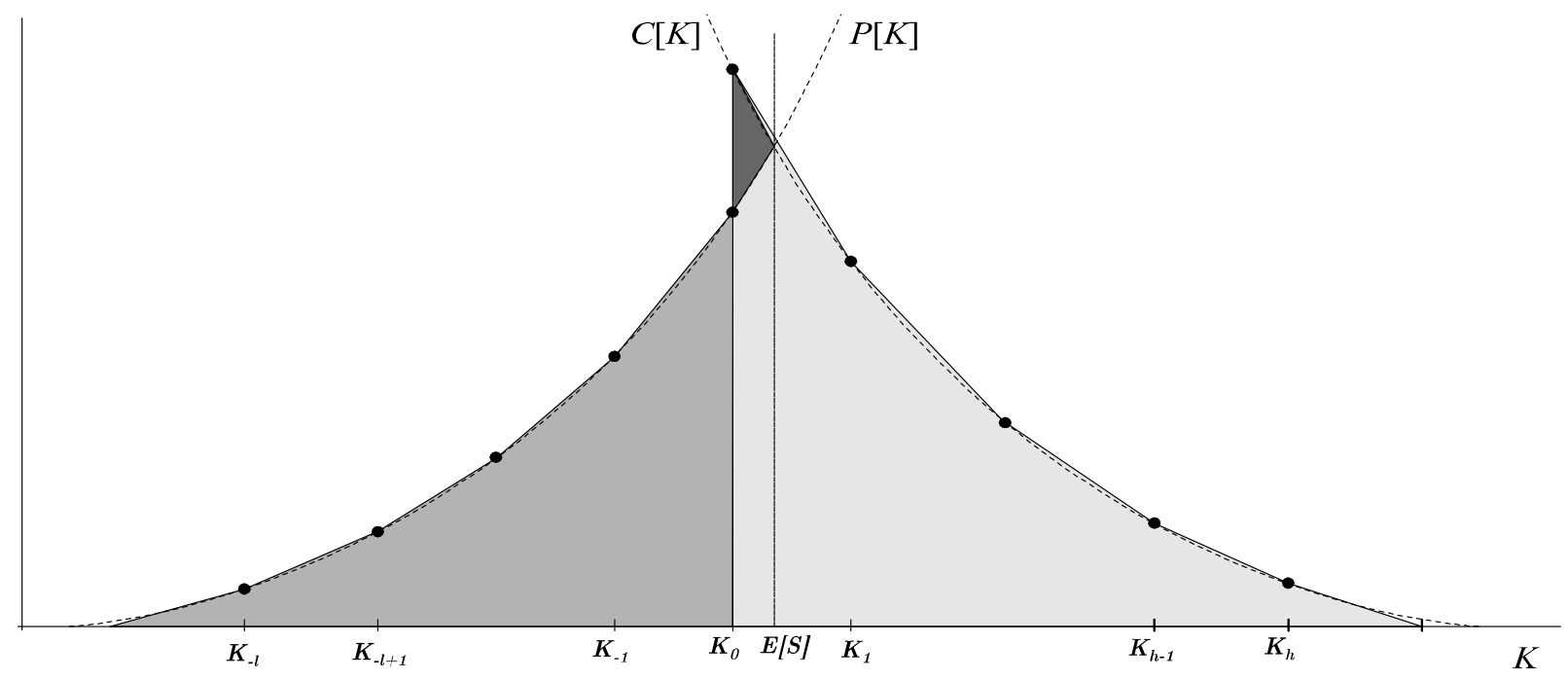

Figure 5: Exact and approximate value of $\frac{\mathrm{e}^{-r T}}{2} \operatorname{Var}[S]$.

Taking into account the put-call parity (13), we find that the third integral in the expression for $\frac{\mathrm{e}^{-r T}}{2} \operatorname{Var}[S]$ is given by

$$
I_{3}=-\frac{\mathrm{e}^{-r T}}{2}\left(\mathrm{E}[S]-K_{0}\right)^{2} .
$$

Adding $I_{1}, I_{2}$ and $I_{3}$ and assuming that $K_{1}-K_{0}=K_{0}-K_{-1}$ leads to the approximate expression (19) for $\frac{\mathrm{e}^{-r T}}{2} \operatorname{Var}[S]$.

\section{References}

Carr, P. and Madan, D. (2001), Towards a theory of volatility trading, in 'Option Pricing, Interest Rates and Risk Management', Cambridge University Press, pp. 458-476.

Carr, P. and Wu, L. (2006), 'A tale of two indices', The Journal of Derivatives 13(3), 1329.

Chen, X., Deelstra, G., Dhaene, J. and Vanmaele, M. (2008), 'Static super-replicating strategies for a class of exotic options', Insurance: Mathematics $\&$ Economics 42(3), 1067-1085.

Cheung, K. C. (2010), 'Characterizing a comonotonic random vector by the distribution of the sum of its components', Insurance: Mathematics E Economics 47(2), 130 - 136.

Chicago Board Options Exchange (2009), The CBOE volatility index - VIX, White Paper.

Deelstra, G., Dhaene, J. and Vanmaele, M. (2010), An overview of comonotonicity and its applications in finance and insurance, in B. Oksendal and G. Nunno, eds, 'Advanced Mathematical Methods for Finance', Springer, Germany (Heidelberg). 
Dhaene, J., Denuit, M., Goovaerts, M., Kaas, R. and Vyncke, D. (2002a), 'The concept of comonotonicity in actuarial science and finance: theory', Insurance: Mathematics $\&$ Economics 31(1), 3-33.

Dhaene, J., Denuit, M., Goovaerts, M., Kaas, R. and Vyncke, D. (2002b), 'The concept of comonotonicity in actuarial science and finance: applications', Insurance: Mathematics E Economics 31(1), 133-161.

Dhaene, J., Dony, J., Forys, M. B., Linders, D. and Schoutens, W. (2011), 'FIX - The Fear Index: Measuring Market Fear', SSRN eLibrary .

Dhaene, J. and Kukush, A. (2010), Comonotonic prices in arbitrage-free markets, Working Paper, Leuven: K.U.Leuven - Faculty of Business and Economics.

Embrechts, P., McNeil, A. and Straumann, D. (1999), Correlation and dependence in risk management: properties and pitfalls, in 'Risk Management: Value at Risk and Beyond', Cambridge University Press, pp. 176-223.

Harmon, D., De Aguiar, M. A., Chinellato, D. D., Braha, D., Epstein, I. and Bar-Yam, Y. (2011), 'Predicting Economic Market Crises Using Measures of Collective Panic', SSRN eLibrary .

Hobson, D., Laurence, P. and Wang, T. (2005), 'Static-arbitrage upper bounds for the prices of basket options', Quantitative Finance 5(4), 329-342.

Laurence, P. (2007), Hedging and pricing of generalized spread options and the market implied comonotonicity gap, Workshop and Mid-Term Conference on Advanced Mathematical Methods for Finance, Vienna University, September 2007.

Laurence, P. and Wang, T.-H. (2008), 'Distribution-free upper bounds for spread options and market-implied antimonotonicity gap', The European Journal of Finance 14(8), 717 -734 .

Linders, D., Dhaene, J. and Schoutens, W. (2011), Model-free bounds for basket call and put options: a unified approach, FBE research report, Leuven: K.U.Leuven - Faculty of Business and Economics. 